ARTICLE

https://doi.org/10.1038/s41467-019-11265-y

\title{
Role of p110a subunit of PI3-kinase in skeletal muscle mitochondrial homeostasis and metabolism
}

\author{
Mengyao Ella Li', Hans P.M.M. Lauritzen ${ }^{1}$, Brian T. O'Neill ${ }^{1,2}$, Chih-Hao Wang ${ }^{1}$, Weikang Cai ${ }^{1}$, Bruna B. Brandao ${ }^{1}$, \\ Masaji Sakaguchi ${ }^{1}$, Rongya Tao ${ }^{3}$, Michael F. Hirshman ${ }^{1}$, Samir Softic ${ }^{1,4} \&$ C. Ronald Kahn ${ }^{1}$
}

Skeletal muscle insulin resistance, decreased phosphatidylinositol 3-kinase (PI3K) activation and altered mitochondrial function are hallmarks of type 2 diabetes. To determine the relationship between these abnormalities, we created mice with muscle-specific knockout of the p110 $\alpha$ or p110 $\beta$ catalytic subunits of PI3K. We find that mice with muscle-specific knockout of $p 110 \alpha$, but not p110 $\beta$, display impaired insulin signaling and reduced muscle size due to enhanced proteasomal and autophagic activity. Despite insulin resistance and muscle atro-

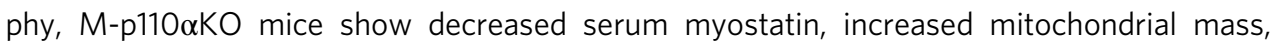
increased mitochondrial fusion, and increased PGC1 $\alpha$ expression, especially PCG1 $\alpha 2$ and PCG1 3 3. This leads to enhanced mitochondrial oxidative capacity, increased muscle NADH content, and higher muscle free radical release measured in vivo using pMitoTimer reporter. Thus, p110 $\alpha$ is the dominant catalytic isoform of PI3K in muscle in control of insulin sensitivity and muscle mass, and has a unique role in mitochondrial homeostasis in skeletal muscle.

\footnotetext{
${ }^{1}$ Section of Integrative Physiology and Metabolism, Joslin Diabetes Center, Harvard Medical School, Boston, MA 02215, USA. ${ }^{2}$ Division of Endocrinology and Metabolism, Fraternal Order of Eagles Diabetes Research Center, University of lowa Carver College of Medicine, lowa City, IA 52242, USA. ${ }^{3}$ Division of Endocrinology, Children's Hospital Boston, Harvard Medical School, Boston, MA 02115, USA. ${ }^{4}$ Division of Gastroenterology, Hepatology and Nutrition, Children's Hospital Boston, Harvard Medical School, Boston, MA 02115, USA. Correspondence and requests for materials should be addressed to C.R.K. (email: C.Ronald.Kahn@joslin.harvard.edu)
} 
P hosphatidylinositol 3-kinase (PI3K) is critical in insulin action and plays key regulatory roles in cell metabolism ${ }^{1}$. Class IA PI3Ks are heterodimers consisting of a regulatory subunit (usually p85a/ $\beta$ ), and a catalytic subunit (usually p110 $\alpha$, $\mathrm{p} 110 \beta$, and $\mathrm{p} 110 \delta$ ) that catalyzes the production of phosphatidylinositol 3,4,5-trisphosphate (PIP3) in response to ligand stimulation ${ }^{1}$. Studies with tissue-specific knockout and chemical inhibitors have shown different roles of the major catalytic subunits, $\mathrm{p} 110 \alpha$ and $\mathrm{p} 110 \beta$, in insulin action in the liver and fat ${ }^{2-4}$. Thus, while impaired glucose tolerance is observed in mice with liver-specific knockout of either $\mathrm{p} 110 \alpha$ or $\mathrm{p} 110 \beta$, only liverspecific deletion of $\mathrm{p} 110 \alpha$ results in a blunting of insulinstimulated Akt phosphorylation ${ }^{2,3}$. Similarly, loss of p110a, but not $\mathrm{p} 110 \beta$, in adipose tissue results in glucose intolerance, despite no reduction in fat mass ${ }^{4}$. These data indicate that while both p110 $\alpha$ and p110 $\beta$ catalytic subunits are present in most insulinsensitive tissues, in the liver and fat, p110 a appears to be more important in metabolic response through the PI3K-AKT axis.

Skeletal muscle insulin resistance is a hallmark of type 2 diabetes mellitus and the obese-prediabetic state ${ }^{5}$. This is usually associated with mitochondrial dysfunction manifest by decreased mitochondrial content and mitochondrial oxidative capacity ${ }^{6}$, although some studies have found no change or even increased mitochondrial metabolism in obese and insulin-resistant humans and rodents ${ }^{7,8}$, indicating a complex relationship between mitochondrial function and insulin action. In addition, diabetes and insulin resistance have been associated with altered muscle growth and fiber type ${ }^{9}$, and muscle wasting has been shown to correlate with defects in insulin and IGF-1 signaling in skeletal muscle ${ }^{9,10}$. Defects in insulin stimulation of PI3K in muscle have also been detected in type 2 diabetes, obesity, and even prediabetes ${ }^{11,12}$. The roles the $\mathrm{p} 110 \alpha$ and $\mathrm{p} 110 \beta$ catalytic subunits in regulation of muscle metabolism, however, are not known.

The aim of this study was to define the relative roles of the $\mathrm{p} 110 \alpha$ and $\mathrm{p} 110 \beta$ isoforms in the regulation of muscle growth and mitochondrial metabolism. We find that the loss of p110a, but not $\mathrm{p} 110 \beta$, in muscle leads to insulin resistance and reduced muscle mass primarily due to enhanced protein degradation. Interestingly, loss of $\mathrm{p} 110 \alpha$ is also accompanied by increased expression of PGC1 $\alpha$, especially the $\underline{\alpha 2}$ and $\underline{\alpha 3}$ isoforms, increased mitochondrial biogenesis, altered mitochondrial dynamics, and increased mitochondrial oxidative capacity. Taken together, these data indicate that PI $3 \mathrm{~K}$ and the $\mathrm{p} 110 \mathrm{a}$ catalytic subunit play a unique role in regulation of muscle metabolism, being critical for maintenance of muscle mass and normal mitochondrial homeostasis. This bifunctional role of p110a may, in part, explain the association between muscle insulin resistance and mitochondrial dysfunction in diabetes and other insulin-resistant states.

\section{Results}

Decreased PI3K activity and impaired insulin signaling. Skeletal muscle expresses both major catalytic subunits of PI3K. RNA-Seq analysis of muscle revealed that $p 110 \alpha$ mRNA was 12 -fold higher than $p 110 \beta$ mRNA when analyzed as the fraction of total reads (Supplementary Fig. 1a), suggesting a dominant role of $\mathrm{p} 110 \alpha$ in this tissue. To more directly address this question, we specifically inactivated the $p 110 \alpha$ or $p 110 \beta$ genes in skeletal muscle using Cre-lox recombination. The resultant Mp $110 \alpha \mathrm{KO}$ displayed a $77 \%$ and a $83 \%$ reduction in $p 110 \alpha$ mRNA and protein levels in pooled hindlimb skeletal muscles, respectively (Supplementary Fig. 1b, c). This percentage approximates the relative contribution of myocytes to other cells in skeletal muscle, such as satellite cells, adipocytes, endothelial cells, nerves, and fibroblasts ${ }^{13}$. Although no good antibodies exist for $\mathrm{p} 110 \beta$ protein, there was a similar $79 \%$ reduction in $p 110 \beta$ mRNA in skeletal muscle of M-p110 $\beta \mathrm{KO}$ (Supplementary Fig. 1d).

Consistent with the relative abundance of $p 110 \alpha$ versus $p 110 \beta$ mRNA, insulin-stimulated PI3K activity in anti-IRS-1 and antipTyr immunoprecipitates was almost completely lost in muscle extracts of M-p110aKO (Fig. 1a), whereas no significant difference in PI3K activity was detected in $\mathrm{M}$-p110 $3 \mathrm{KO}$ mice (Supplementary Fig. 1e), despite a trend toward reduced pTyrassociated PI3K activity in these mice (IP:pTyr, $p=0.12$, Student's $t$ test). Similarly, a qualitative comparison of insulinstimulated PIP3 immunofluorescence-the product of PI3Kshowed almost complete loss of PIP3 staining in muscle of $\mathrm{M}$ p110aKO, whereas the PIP3 level in M-p110ßKO was similar to the controls (Supplementary Fig. 1f, g). As expected, musclespecific deletion of p110a impaired insulin signaling with a $42 \%$ decrease in phosphorylation of AKT in skeletal muscle (Fig. 1b, c). Somewhat surprisingly, following insulin injection, there was a similar decrease in ERK phosphorylation $(p=0.04)$ (Supplementary Fig. 2a) and a tendency toward reduced 4EBP1 phosphorylation (Supplementary Fig. 2b), suggesting some downstream crosstalk between these pathways. However, there was no significant difference in AKT phosphorylation in M-p110aKO, as compared with controls, following 13 weeks of high-fat diet (HFD) feeding (60\% calories from fat) (Supplementary Fig. 2c). ERK phosphorylation was also not different, indicating that insulin resistance induced by HFD was sufficient to abrogate the difference in insulin signaling observed between controls and $\mathrm{M}$ p110aKO on chow diet (CD) (Supplementary Fig. 2c). By comparison, no changes were displayed in the phosphorylation levels of AKT, ERK, GSK3 $\beta$, or 4EBP1 in M-p110 $3 \mathrm{KO}$, whether on CD or HFD (Supplementary Fig. 2d, e).

Increased proteasome activity and autophagy signalings. Muscle gene expression was assessed using RNA-Seq analysis. Principle component analysis (PCA) revealed distinct gene expression patterns in skeletal muscles of controls and $\mathrm{M}$ p110aKO, both in fed and fasted states (Fig. 1d). Unsupervised KEGG analysis revealed several pathways that were significantly enriched in $\mathrm{M}$-p110aKO, including genes involved in the TCA cycle (Fig. 1e), oxidative phosphorylation (OXPHOS) (Fig. 1f) and lysosome/autophagosome signaling (Fig. 1g). Interestingly, in both TCA cycle and OXPHOS gene sets, discrete subsets of genes were upregulated in fed versus fasted state, suggesting the differences in metabolic adaptation to a state of energy deprivation.

Autophagy is an important pathway that regulates protein degradation in muscle and is regulated by FoxO transcriptional factors ${ }^{14,15}$. Increased autophagy markers were displayed in quadriceps muscle of M-p110aKO, with $53-130 \%$ increases in ULK $1^{555}$ phosphorylation, a nutrient-dependent initiator of autophagy and 3- to 3.9-fold increases in the total LC3B protein, a marker and mediator of autophagy ${ }^{16}$ in both fed and fasted states (Fig. 2a). LC3B levels were not increased with fasting in controls or $\mathrm{M}-\mathrm{p} 110 \alpha \mathrm{KO}$, likely due to increased protein degradation, but were increased in both groups of fasted mice after treatment with colchicine to block autophagic flux. In soleus muscle, LC3B protein levels were less upregulated in fed M-p110aKO, and no difference between the groups was seen with fasting (Supplementary Fig. 2f), suggesting that a difference in autophagy is not as prominent in oxidative fibers. Interestingly, p110a expression was also regulated by energy status in quadriceps muscle of controls with a 2.3 -fold increase in p110a protein after $24 \mathrm{~h}$ of fasting (Fig. 2a). This may explain the different gene expression profile in TA muscle of $\mathrm{M}$ p110aKO between fed and fasted states. Again, an increase in p110a protein upon fasting was not observed in oxidative 
a

$\square$ Lox

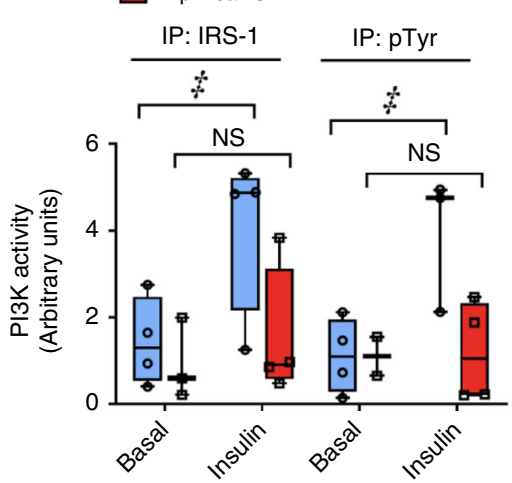

b

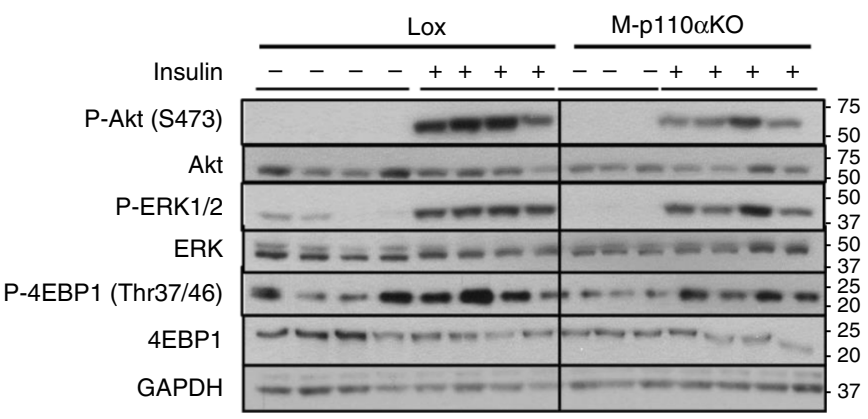

C

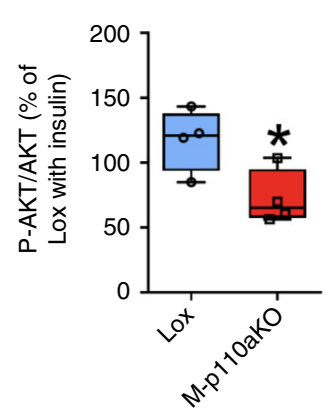

f

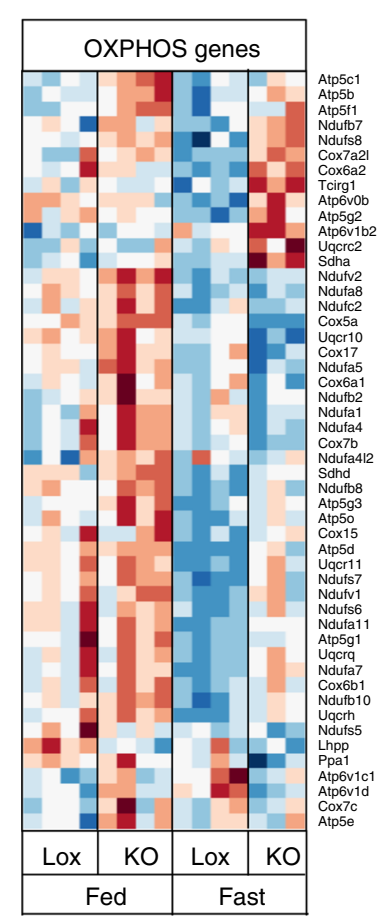

d

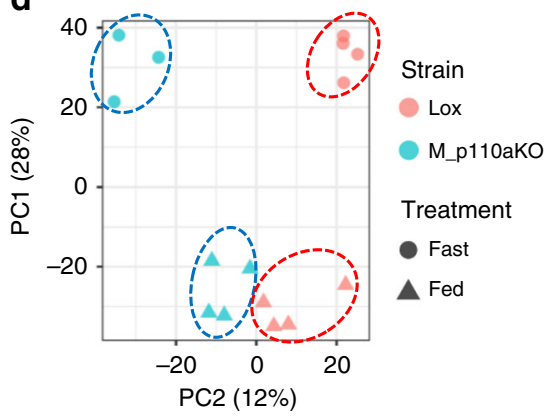

g

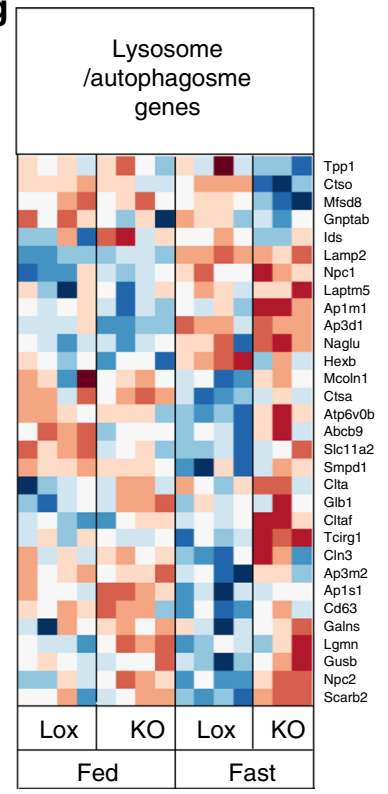

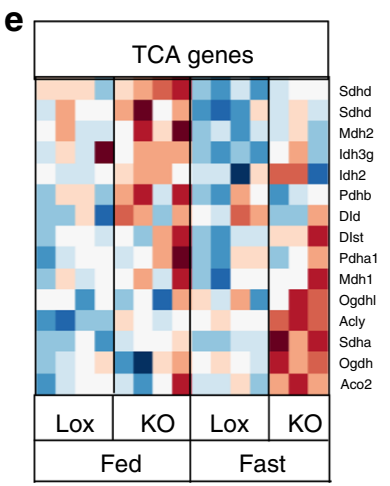

Color key

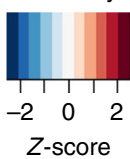

Fig. 1 Impaired insulin signaling and changes in FA oxidation and lysosome pathways. a PI3K activities from extracts immunoprecipitated with anti-IRS-1 or anti-pTyr antibodies in gastrocnemius muscles of M-p110 $\alpha \mathrm{KO}$ and control mice after insulin injection ( $5 \mathrm{U}$ of regular insulin via the inferior vena cava) ( $n=$ 4). b, c Western blot of insulin signaling (b) and densitometric analysis of P-AKT/AKT (c) in quadriceps muscle of M-p110 $\alpha K O$ and controls that were fasted overnight and treated with saline or insulin intravenously. d Principal component analysis (PCA) plot of transcriptomic analysis by RNA-Seq from TA muscles of control and M-p110 $\alpha \mathrm{KO}$ in fed and 24 -h fasted conditions (ctrl, $n=3 ; \mathrm{KO}, n=4$ ). e-g Heatmap of transcripts involved in KEGG pathways analyzed from the RNA-Seq experiment in panel $\mathbf{d}(\mathrm{ctrl}, n=3 ; \mathrm{KO}, n=4)$. Genes involved in the TCA cycle (e), oxidative phosphorylation (f), and autophagosome- lysosme pathways ( $\mathbf{g}$ ) were listed in the heatmap, and the color intensities indicate Z-score of each sample by gene. All mice were 3month old. ${ }^{\star} P<0.05$ by Student's $t$ test; $¥ P<0.05$ by two-way ANOVA. PC1 principle coordinate $1, P C 2$ principle coordinate 2 . Box plots visualize the fivenumber summary of a data set (minimum, lower quartile, median, upper quartile, and maximum) 

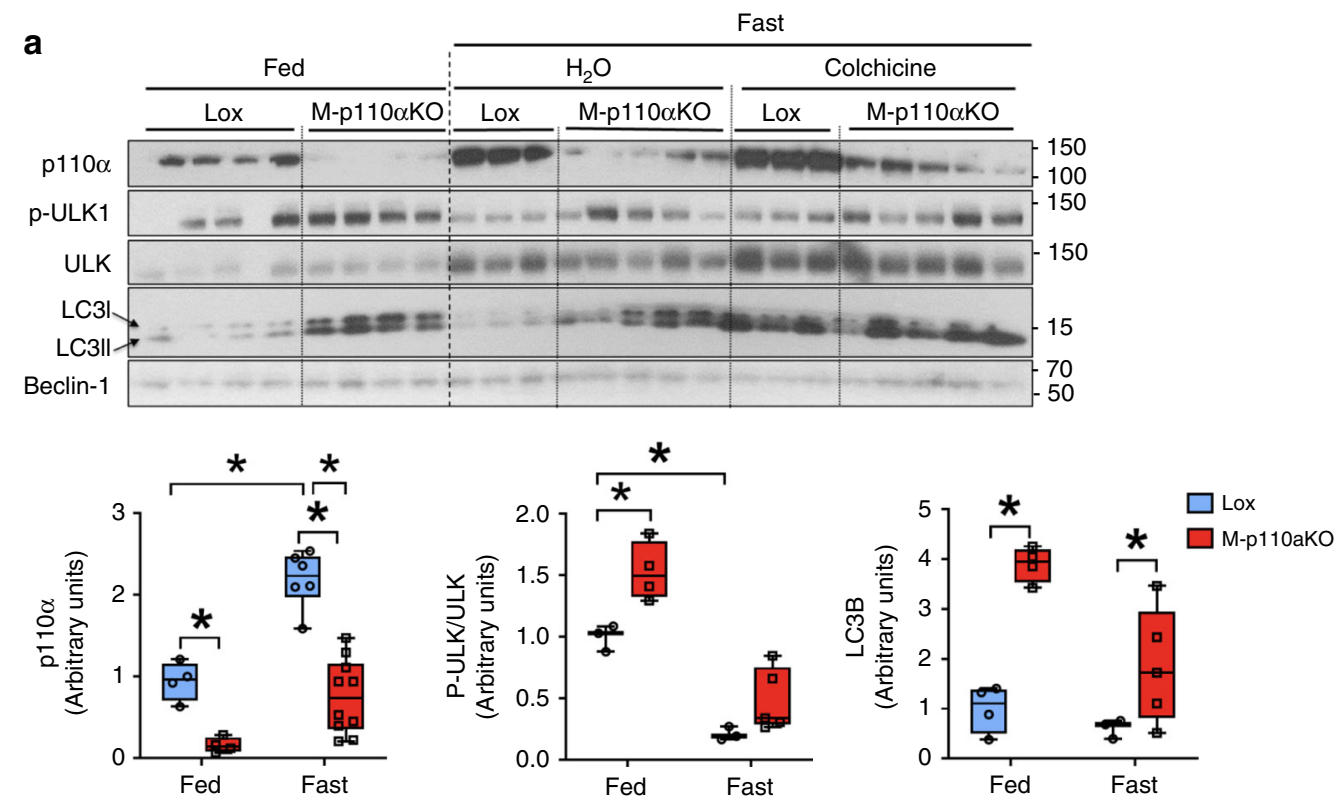

b

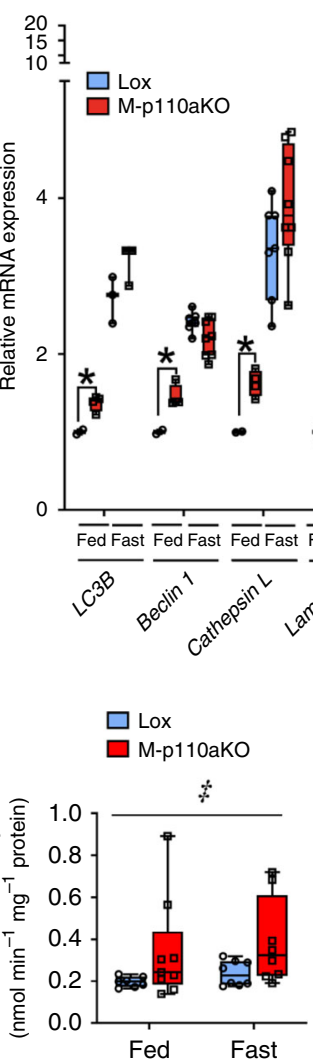

c

Fed

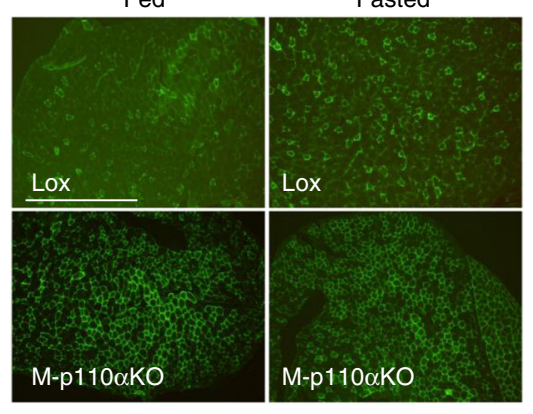

d

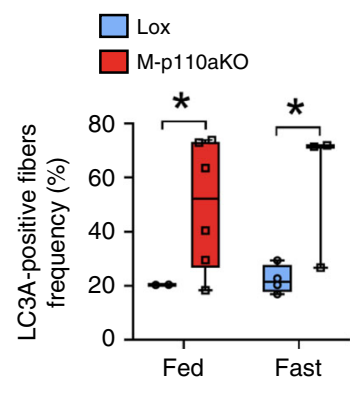

Fig. 2 Increased markers of autophagy-lysosomal degradation and proteasome activity. a Western blot and densitometric analysis of autophagy

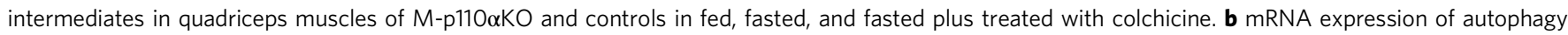
genes in TA muscles of M-p110 $\alpha \mathrm{KO}$ and controls $(n=4)$. c LC3A immunostaining of quadriceps from M-p110 $\alpha$ KO and controls (scale bar $=500 \mu$ m). d Percentage of LC3A-positive fibers per cross-section of quadriceps muscles in (c) ( $n=4-7$ ). e Proteasome activity in gastrocnemius muscles of Mp $110 \alpha \mathrm{KO}$ and controls using a peptidyl glutamyl-like (LLE) substrate $(n=8)$. f Proteolysis rate measured by tyrosine release ex vivo in EDL and soleus muscles isolated from fed control and M-p110 $\alpha \mathrm{KO}$ at 24 months of age (ctrl, $n=5$; KO, $n=6$ ). Mice for Fig. 2a-e were 3-month old, and mice for Fig. 2f were 24 -month old. Mice for Fig. $2 a-e$ were fasted for $24 \mathrm{~h}$ or randomly fed. ${ }^{\star} P<0.05$ by Student's $t$ test; $\$ P<0.05$ by two-way ANOVA. Box plots visualize the five-number summary of the data set (minimum, lower quartile, median, upper quartile, and maximum). The data are mean \pm SEM in bar graph

soleus muscle (Supplementary Fig. 2f). In addition to increases in autophagy markers at the protein level, autophagy regulating genes, such as LC3b, beclin1, and cathepsin L, were also increased $40-63 \%$ at the mRNA level in glycolytic TA muscle in fed state (all $p<0.05$, Student's $t$ test) (Fig. 2b). Qualitative immunostaining of muscle sections confirmed increased percent of LC3-positive fibers in M-p110aKO in both fed and fasted conditions (Fig. 2c, d). 
Increased proteasome activity was also observed in muscle extracts of M-p110aKO in both fed (72\%) and fasted (62\%) conditions using peptidyl glutamyl-like (LLE) substrates (Fig. 2e). This occurred with no change in mRNA expression of genes that encode proteasome subunit proteins (Supplementary Fig. 3a), suggesting increased proteasome activity may be induced by a post-transcriptional or conformational change in the protein ${ }^{17}$. Proteolysis demonstrated a $19 \%$ increase in tyrosine release from extensor digitorum longus (EDL), but not soleus muscle, in $\mathrm{M}$ p1 $10 \alpha \mathrm{KO}$ at 24 months of age ( $p<0.05$, Student's $t$ test) (Fig. 2f), indicating muscle-specific increases in proteolysis at an age generally accompanied with muscle wasting ${ }^{18}$. These changes were not observed, however, in M-p110aKO at 5 months of age, when there was minimal loss of muscle mass (Supplementary Fig. 3b). Together these data indicate that both the proteasome pathway and the autophagy pathway were upregulated in $\mathrm{M}$ p110aKO.

Forkhead box $\mathrm{O}$ (FoxO) transcription factors are downstream targets of the PI3K-AKT axis and regulate skeletal muscle mass through both proteasome and autophagy pathways ${ }^{15,19,20}$. In the absence of AKT-mediated phosphorylation, FoxO proteins are translocated from the cytosol to the nucleus where they upregulate expression of atrophy-related genes. In spite of decreased AKT activation/phosphorylation, there was no difference in FoxO nuclear translocation in $\mathrm{M}-\mathrm{p} 110 \alpha \mathrm{KO}$ and controls (Supplementary Fig. 3c).

In summary, loss of p110a, but not p110 3 , leads to decreased insulin signaling as documented by decreased PI3K activity, reduced PIP3 levels, and diminished AKT phosphorylation. This leads to increased autophagy and proteasome degradation in glycolytic and mixed muscle fibers, resulting in increased muscle atrophy with aging. These results are in agreement with previous studies from our lab reporting that a loss of insulin signaling in muscle in muscle-specific IR and IGF1R knockout mice leads to increased muscle atrophy ${ }^{10}$.

Larger mitochondria due to altered mitochondrial dynamics. Ultrastructure of the mitochondria was assessed in transverse sections of EDL and soleus muscles by electron microscopy (EM). In glycolytic EDL muscle, M-p110aKO displayed an $83 \%$ increase of average mitochondrial area compared with controls, whereas there was no difference in the more oxidative soleus muscle (Fig. 3a, b). This occurred with no change in the mitochondrial number in either EDL or soleus muscles in M-p110aKO (Supplementary Fig. 4a). In control mice, average mitochondrial area was $\sim 90 \%$ greater in soleus than EDL muscle, reflecting more slow, oxidative fibers in the soleus compared with EDL.

To directly assess the effect of muscle-specific p110a deletion on mitochondrial morphology in vivo, we transfected a mito-GFP plasmid that specifically targets the mitochondrial matrix ${ }^{21}$ into quadriceps muscles of living mice using a gene gun delivery system $^{22}$. Quantitation using confocal microscopy showed that the total mitochondrial area (Fig. 3c) and the average size of a single mitochondrion (Fig. $3 \mathrm{~d}$ ) were increased by 2 - to 2.5 -fold in M-p110aKO mice. The increase in the total mitochondrial area was further accentuated by a decrease in the muscle fiber size as intravital imaging also confirmed a $17 \%$ decrease of the muscle fiber area, as quantified from intramyofibrillar images in $\mathrm{M}$ p110aKO mice (Supplementary Fig. 4b).

Mitochondrial fusion and fission regulate mitochondrial morphology and play important roles in mitochondrial quality control $^{23}$. Optic atrophy 1 (OPA1) and mitofusin 2 (Mfn2) are proteins that drive mitochondrial inner- and outer membrane fusion $^{24}$, and their protein levels were increased by $36 \%$ and $38 \%$ in TA muscle of fed $\mathrm{M}-\mathrm{p} 110 \alpha \mathrm{KO}$, respectively (all $p<0.05$,
Student's $t$ test) (Fig. 3e). Dynamin-related protein 1 (Drp1) is a driver of mitochondrial fission and is translocated from the cytosol to mitochondrial membranes upon dephosphorylation of Ser637 25 . Previous studies have shown that during fasting, mitochondrial fission is inhibited to sustain the ATP demand during nutrient deprivation ${ }^{26}$. In fed state, M-p110aKO had a $50 \%$ increase in Drp1 phosphorylation at Ser637 compared with controls $(p<0.05$, Student's $t$ test) (Fig. 3e), creating a decreased drive for mitochondrial fission in these mice. Thus, loss of p110a in muscle shifts mitochondrial dynamic toward an increased fusion and decreased fission, resulting in larger and more elongated mitochondria in M-p110aKO.

Increased mitochondrial biogenesis in M-p110aKO. The levels of mitochondrial- and nuclear-encoded proteins involved in OXPHOS were assessed using muscle extracts. In fed state, loss of muscle p110a led to a $69 \%$ increase in protein levels of NDUFB8 in Complex I, and a $280 \%$ increase in succinate dehydrogenase subunit B (SDHB) from Complex II (Fig. 4a, b). Upon $24 \mathrm{~h}$ of fasting, M-p110aKO displayed further increases of mitochondrial protein expression, including SDHB (about 200\%), ubiquinol cytochrome $c$ reductase core protein 2 (UQCRC2) of Complex III (86\%), and ATP5A of Complex V (50\%) (all $p<0.05$, Student's $t$ test). These changes in mitochondrial proteins were accompanied by increase in mitochondrial respiratory enzyme activities in muscle of $\mathrm{M}-\mathrm{p} 110 \mathrm{aKO}$, with a $14 \%$ increase in Complex IV activity $(p<0.05$, Student's $t$ test) (Fig. $4 c)$ and tendencies of increased activities of Complexes I to III and II to III in the mitochondria of fasted M-p110aKO (Supplementary Fig. 4c, d). Citrate synthase (CS) catalyzes the first step in the TCA cycle, and is a marker for the intact mitochondria ${ }^{27}$. Loss of p110a in muscle led to a $45 \%$ increase in CS protein expression (Fig. $4 \mathrm{~d}$ ) and a parallel increase in enzyme activity in M-p110aKO in fed and fast states (Fig. 4e).

PGC1al is a transcription factor that controls oxidative metabolism in muscle and other tissues ${ }^{28}$. Four transcripts of $P G C 1 \alpha(1,2,3$, and 4) are produced from PGC1 $\alpha$ gene and regulate the expression of different downstream genes ${ }^{29}$. PGC1a1 functions as a major regulator of mitochondrial biogenesis, whereas PGC1a4 is mostly known to promote muscle hypertrophy ${ }^{30}$. Little is known about the functions of PGC1a2 and PGC1a3. Muscle-specific deletion of p110a led to increased mRNA expression of all PGC1 $\alpha$ isoforms, with $45-55 \%$ increases of PGC1 $\alpha 1$ and $P G C 1 \alpha 4$ and $70-80 \%$ increases in expression of $P G C 1 \alpha 2$ and $P G C 1 \alpha 3$ (all $p<0.05$, Student's $t$ test) (Fig. $4 \mathrm{f}$ ). Upon fasting, mRNA levels of PGC1 $\alpha 1$ and 4 were unchanged, while $P G C 1 \alpha 2$ and 3 were increased by 3.3- and 2.8-fold in controls and further increased in M-p110aKO by three- to fivefold.

Increased mitochondrial oxidative capacity in M-p110aKO. Our findings of increased mitochondrial mass and biogenesis prompted us to measure mitochondrial respiratory capacity and ATP content. Consistent with the increased mitochondrial mass and increased mitochondrial function, ATP levels were increased by $26 \%$ in quadriceps muscle of $\mathrm{M}$-p110aKO compared with controls (Fig. 5a). This was accompanied by a $96 \%$ increase in fatty acid oxidation in the mitochondria isolated from hindlimb muscle of M-p110aKO (Fig. 5b). In addition, there was a $93 \%$ increase in oxygen consumption rate (OCR) in presence of ADP (state 3) with pyruvate/malate as substrates in mitochondria isolated from skeletal muscle of $\mathrm{M}$-p1 $10 \mathrm{\alpha KO}$, indicating enhanced oxidative capacity (Fig. 5c). These changes occurred with no changes in mRNA expression of genes involved in fatty acid oxidation in skeletal muscle of M-p110aKO (Supplementary Fig. 4e). 
a

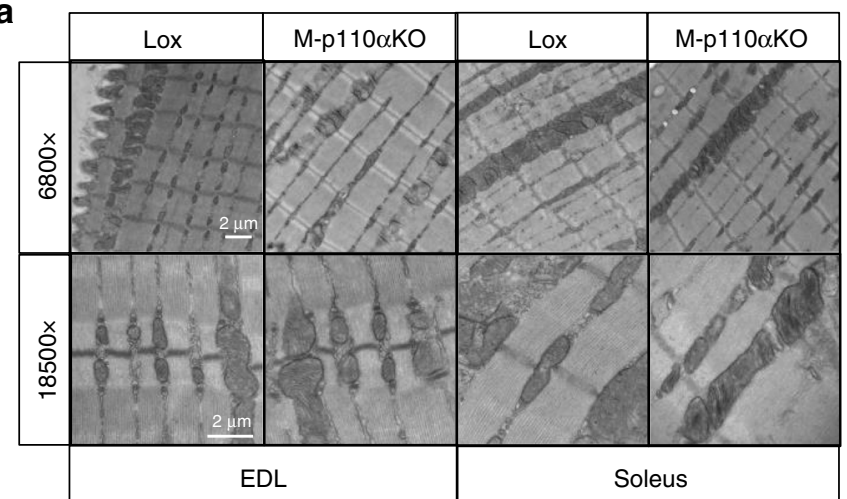

C

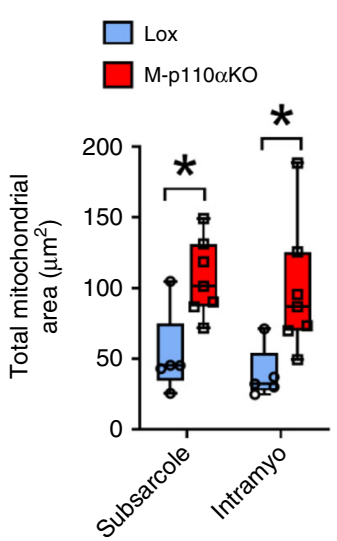

d $\square$ Lox

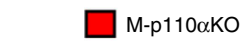

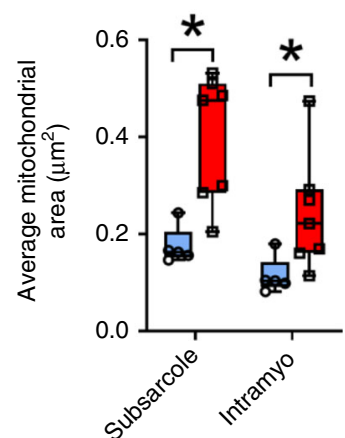

b

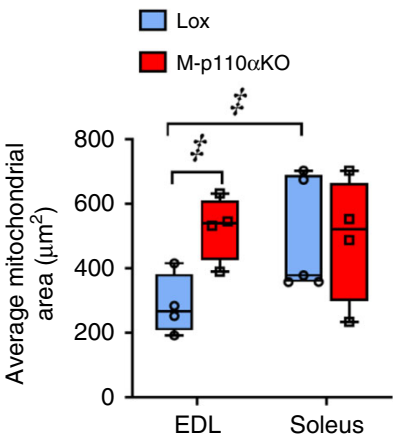

e
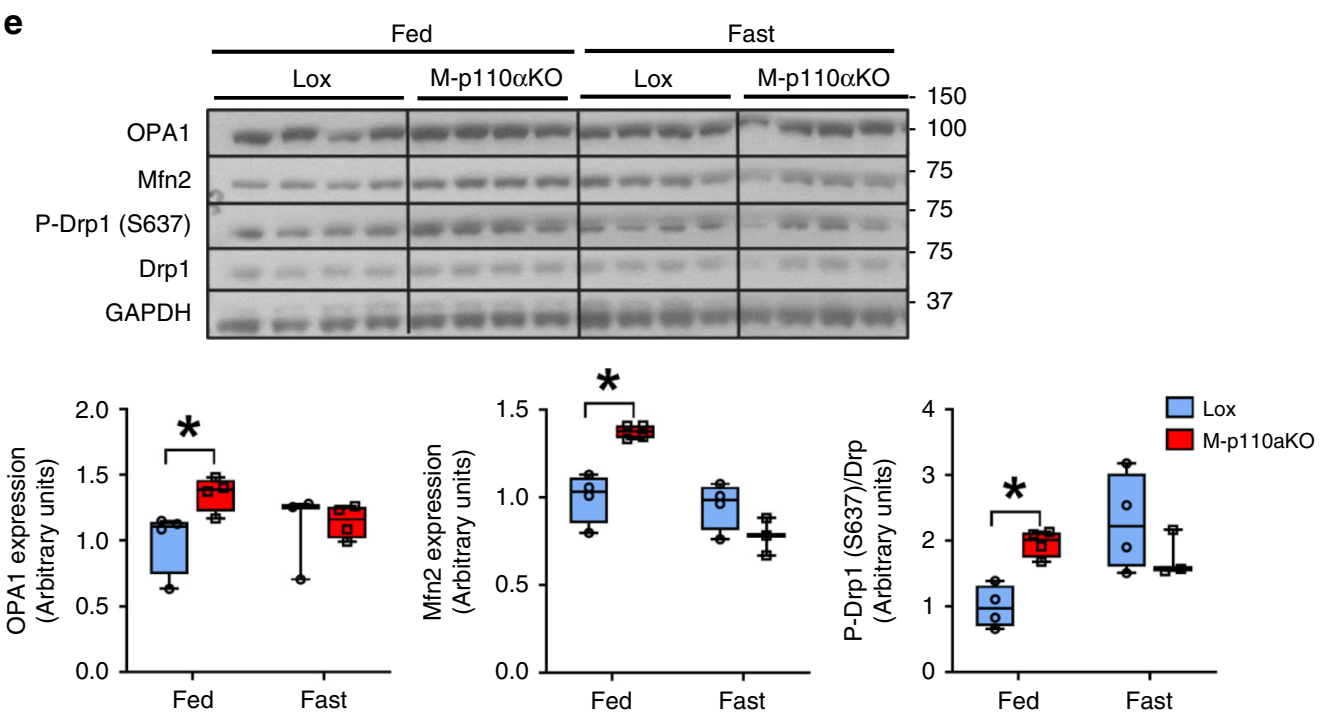

Fig. 3 Larger mitochondria associated with altered mitochondrial dynamics. a Ultrastructure of the mitochondria in transverse planes of EDL and soleus

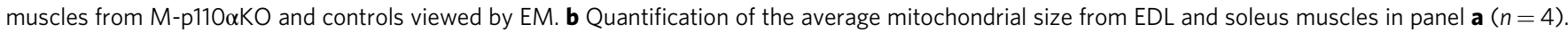
c, $\mathbf{d}$ Quantification of the total mitochondria area per muscle fiber view-sight (c) and the average mitochondrial area (d) from M-p110 $\alpha \mathrm{KO}$ and controls transfected with Mito-GFP in panel c (ctrl, $n=3 ; \mathrm{KO}, n=4$ with four to ten fibers per mouse). e Western blot and densitometric analysis of proteins involved in mitochondrial dynamics in TA muscles of $\mathrm{M}$-p110 $\alpha \mathrm{KO}$ and controls fasted for $24 \mathrm{~h}$ or randomly fed. All mice were 4 - to 5 -month old. ${ }^{\star} P<0.05$ by Student's $t$ test; $\$ P<0.05$ by two-way ANOVA. Electron microscopy EM, subsarcole subsarcolemma, intramyo intramyofibrillar. Box plots visualize the five-number summary of the data set (minimum, lower quartile, median, upper quartile, and maximum)

$\mathrm{NADH}$ is a major product of the TCA cycle, and can be visualized in muscle of living mice by its autofluorescence ${ }^{31}$. In muscle of control mice, NADH autofluorescence was 77\% lower in intramyofibrillar regions compared with those in subsarcolemmal regions (Fig. 5d), consistent with other studies showing lower mitochondrial function in the intramyofibrillar mitochondria ${ }^{32}$. Compared with controls, NADH levels were increased in both intramyofibrillar ( 8-fold) and subsarcolemmal ( 13.6-fold) locations in M-p110aKO, consistent with higher TCA cycle activity. This increased mitochondrial metabolic capacity was associated with increased free radical production in $\mathrm{M}$-p110aKO with $55-60 \%$ increases in combined reactive 
a

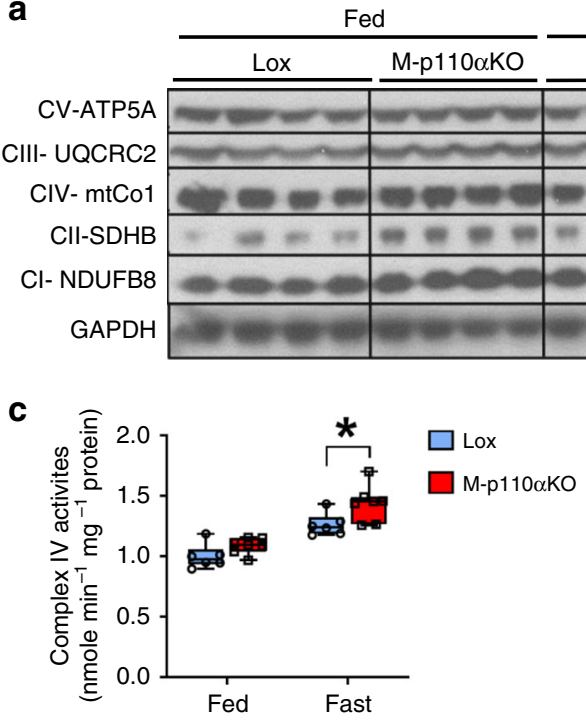

b

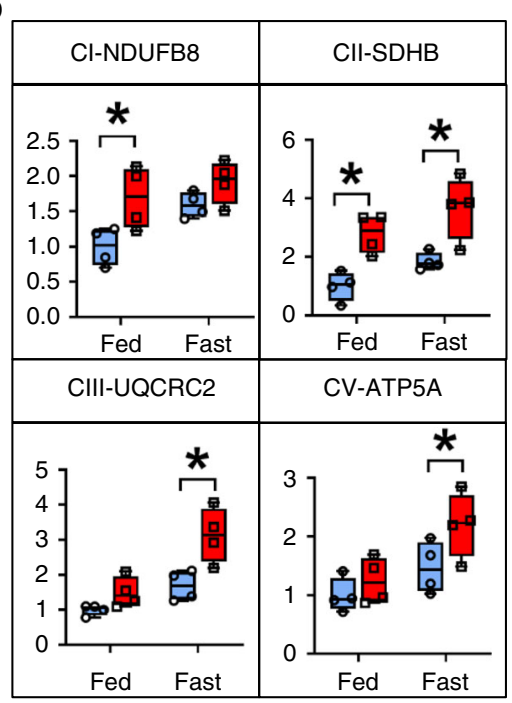

d

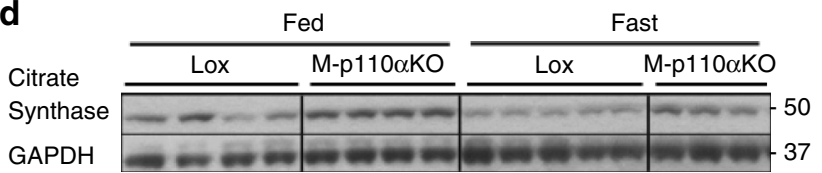

f
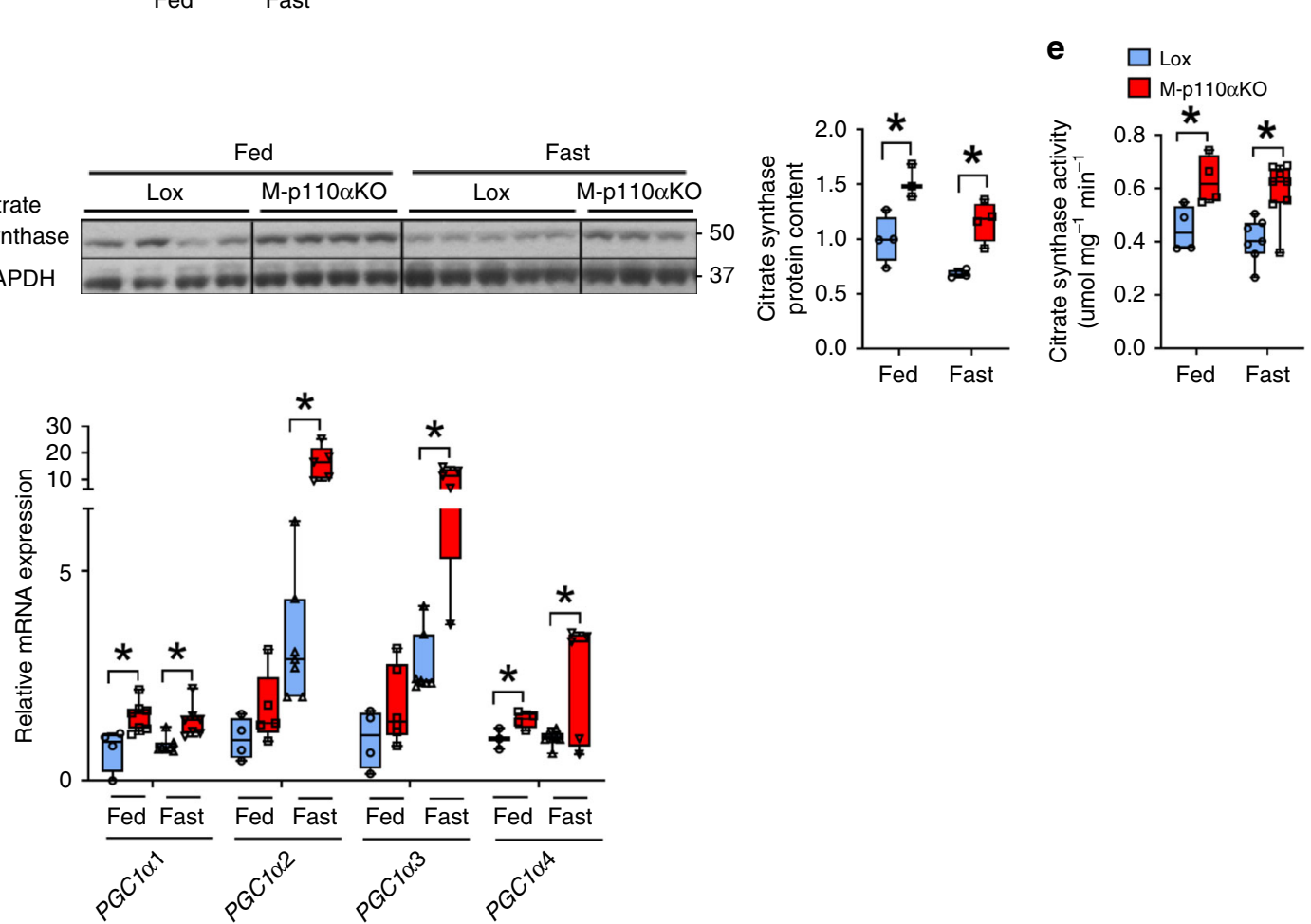

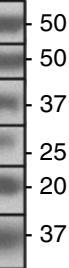

Fed Fast 
a

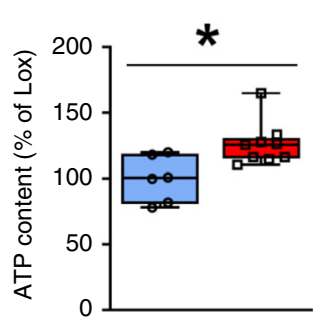

b

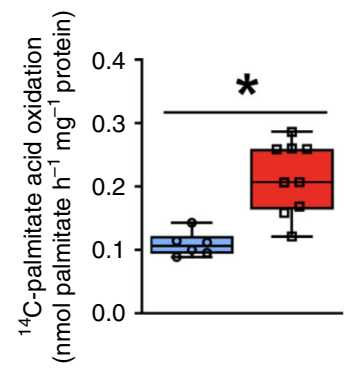

C $\square$ Lox

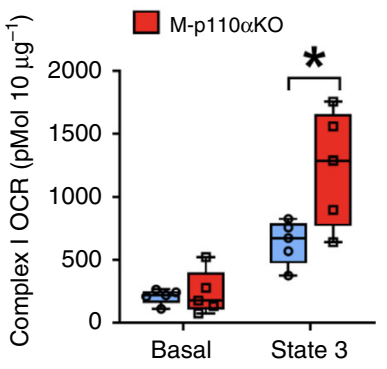

d
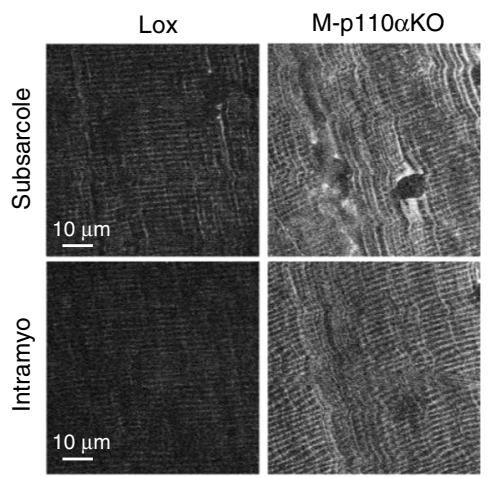

f
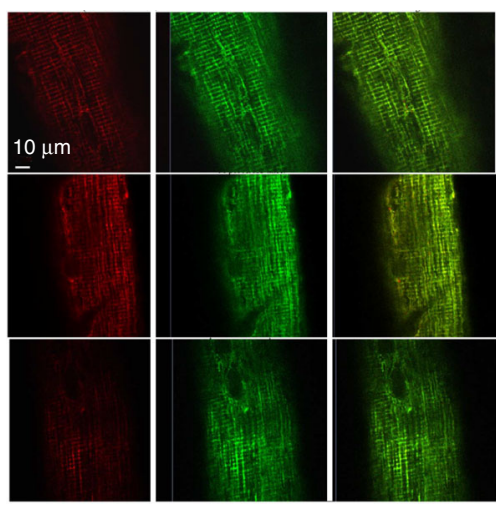

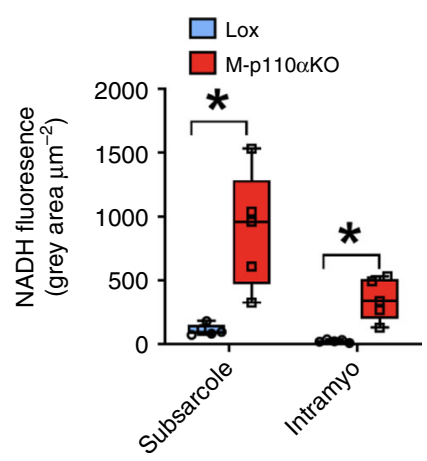

e
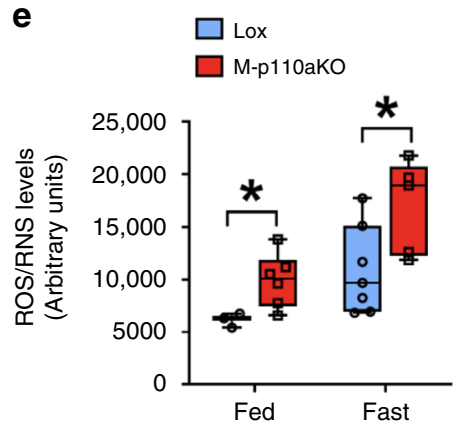

g

Lox
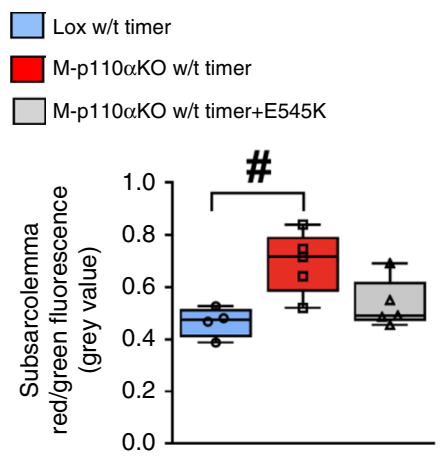

Fig. 5 Increased mitochondrial oxidative capacity in skeletal muscle of M-p110 $\alpha$ KO. a ATP content in quadriceps muscles of M-p110 $\alpha K O$ and control mice in fed state $(\mathrm{ctrl}, n=8 ; \mathrm{KO}, n=9)$. $\mathbf{b}$ Fatty acid oxidation was determined by measuring ${ }^{14} \mathrm{CO}_{2}$ production in the mitochondria isolated from hindlimb of overnight fasted mice using ${ }^{14} \mathrm{C}$-palmitate acid as a substrate (ctrl, $n=7 ; \mathrm{KO}, n=9$ ). c Basal and ADP-dependent (State 3) respiratory rates of the isolated mitochondria from hindlimb of fed $\mathrm{M}-\mathrm{p} 110 \alpha \mathrm{KO}$ and control mice were measured using a Seahorse X24 Flux Analyzer in the presence of a Complex I substrate (pyruvate/malate) (ctrl, $n=5 ; \mathrm{KO}, n=6$ ). $\mathbf{d}$ Intravital multiphoton images of mitochondria-specific NADH autofluorescence in quadriceps muscles of M-p110 $\alpha \mathrm{KO}$ and control mice after overnight fasting (scale bar $=10 \mu \mathrm{m}$ ). e Levels of total free radicals composed of reactive oxygen species (ROS) and reactive nitrogen species (RNS) measured in gastrocnemius muscles of M-p110 $\alpha$ KO and control mice (ctrl, $n=5 ; \mathrm{KO}, n=9$ ). $\mathbf{f}$ pMitoTimer plasmid was in situ transfected into quadriceps muscle fibers of living mice using a gene gun delivery approach and visualized 5 days later by the confocal microscope (upper and middle set of images). A different cohort of M-p110 $\alpha$ KO mice was co-transfected with constitutively active mutant p110 $\alpha$ (PIK3CA) E545K into quadriceps of these mice (bottom set of images). $\mathbf{g}$ Red/green fluorescence in subsarcolemmal position of quadriceps muscles in ( $\mathbf{f}$ ) ( $n=4$ with 3-4 fibers per mouse). All mice were 4 - to 5 -month old. ${ }^{\star} P<0.05$ by Student's $t$ test; ${ }^{\#} P<0.05$ by one-way ANOVA. Subsarco subsarcolemma, intramyo intramyofibrillar, OCR, oxygen consumption rate, Timer pMitoTimer, E545K p110 $\alpha$ (PIK3CA) E545K. Box plots visualize the five-number summary of the data set (minimum, lower quartile, median, upper quartile, and maximum). The data are mean \pm SEM

Hyperinsulinemia and insulin tolerance on HFD and aging. At 3 months of age, M-p110aKO displayed a $60 \%$ increase in serum insulin levels in fed state (Fig. 6a), but intraperitoneal glucosetolerance tests (GTT) revealed no difference between $M$ p110aKO and controls (Fig. 6b). Likewise, insulin-tolerance tests (ITT) were not statistically different, although there was a tendency toward impaired insulin sensitivity in M-p110aKO (Fig. 6c). After 13 weeks on $60 \%$ HFD, both M-p110aKO and controls had 1.5- to 3-fold increases in serum insulin, but no difference was found between the groups (Supplementary Fig. 5a). GTT and ITT were also not different between M-p110aKO and controls on HFD (Supplementary Fig. 5b, c). In general, increased insulin resistance occurs with aging ${ }^{35}$; however, 16-month-old $\mathrm{M}$ p110aKO displayed no difference in whole-body glucose homeostasis compared with controls as measured by intraperitoneal GTT and ITT (Supplementary Fig. 5d, e). Since the whole-body 
a

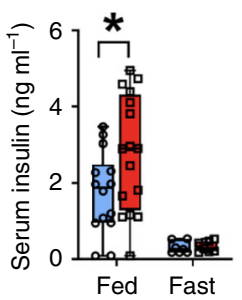

b

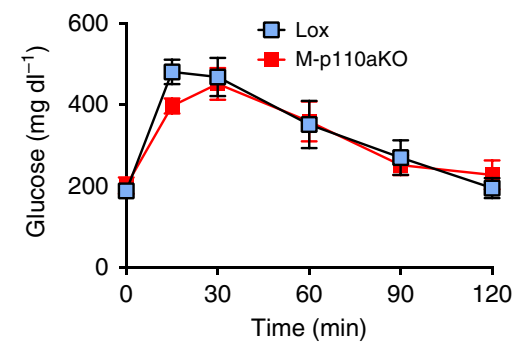

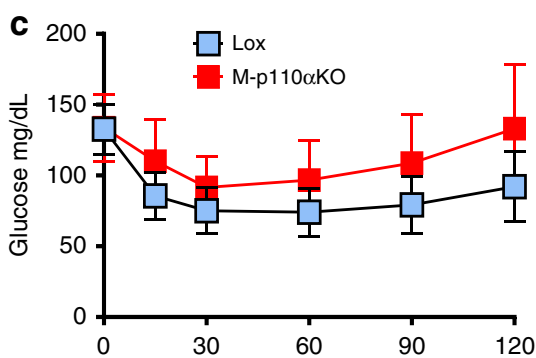

d
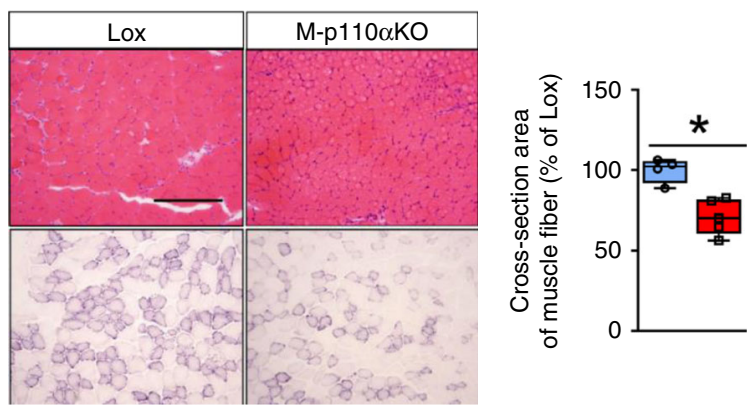
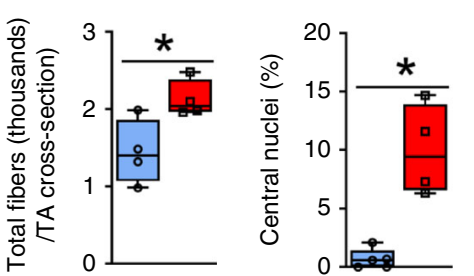

e $\square$ Lox

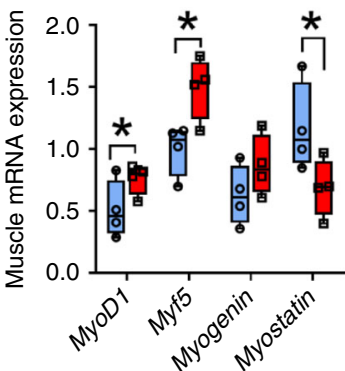

h

i

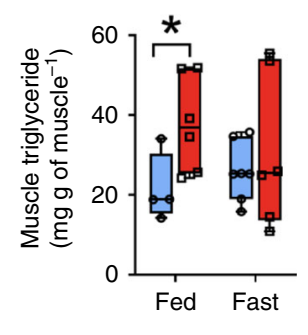

j

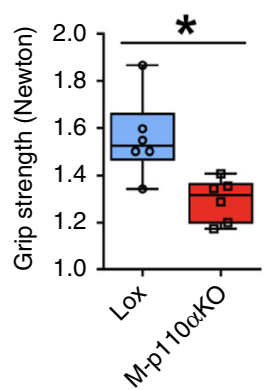

$\mathbf{k}$

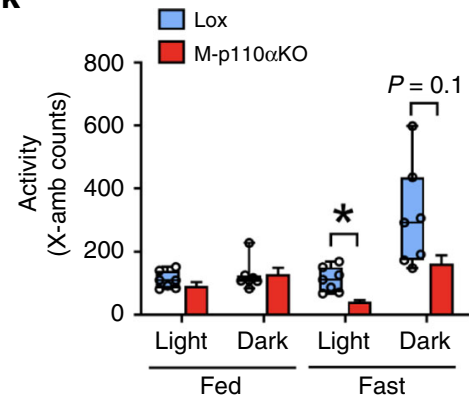

I

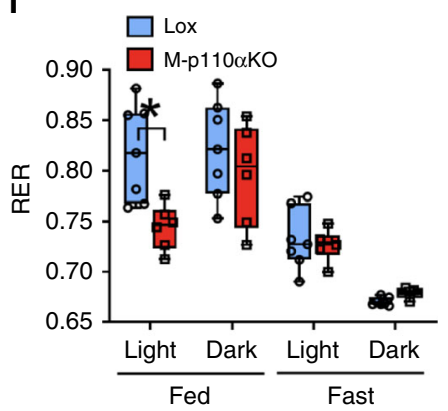

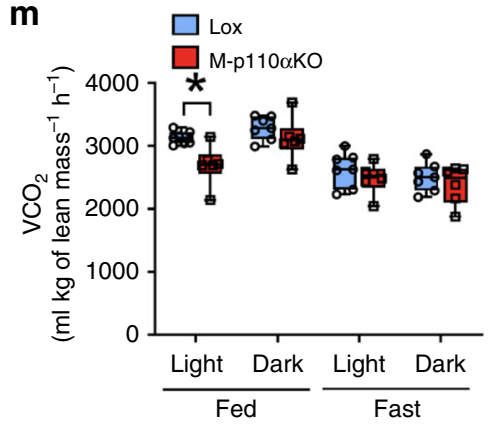

Fig. 6 Hyperinsulinemia, abnormal muscle function, and decreased RER. a Serum insulin levels from M-p110 $\alpha$ KO and control mice fasted for $24 \mathrm{~h}$ or randomly fed at 3 months of age (ctrl, $n=5 ; \mathrm{KO}, n=7)$. b, c Intraperitoneal glucose tolerance test (GTT) and insulin-tolerance test (ITT) (c) performed in

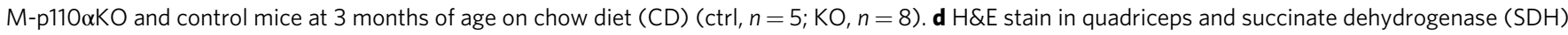
stain in TA muscle cross-sections of M-p110 $\alpha \mathrm{KO}$ and control mice at 3 months of age (scale bar $=200 \mu \mathrm{m}$ ), and quantification of the muscle fiber crosssection area from H\&E stain, total fiber numbers per area of TA muscle cross-section from SDH stain, and percentage of quadriceps myofibers with central nuclei from H\&E stain $(n=5)$. e mRNA expression of myogenic factors in TA muscles from 3-month-old M-p110 $\alpha \mathrm{KO}$ and control mice $(n=5)$. f Serum myostatin levels from 3-month-old M-p110 $\alpha$ KO and control mice fasted for $24 \mathrm{~h}$ or randomly fed (ctrl, $n=5 ; \mathrm{KO}, n=7$ ). $\mathbf{g}$ Percentage of epididymal fat and subcutaneous fat weights per body weight of M-p110 $\mathrm{KKO}$ and control mice at 3 months of age $(n=8)$. $\mathbf{h}$ Triglyceride levels in gastrocnemius muscles of 3-

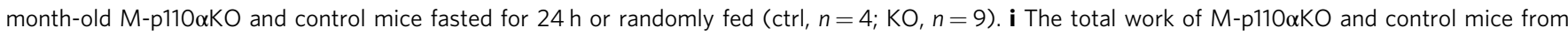
acute treadmill test at 16 months of age $(n=8)$. j Forelimb grip strength from 16-month-old M-p110 $\alpha$ KO and control mice $(n=8)$. k-m Spontaneous activity $(k)$, respiratory exchange ratio (RER) $(\mathrm{I})$, and $\mathrm{VCO}_{2}(\mathrm{~m})$ in 24-month-old $\mathrm{M}$-p110 $\alpha \mathrm{KO}$ and control mice during fed and fast cycles over 48 hrs in CLAMS metabolic cages (ctrl, $n=5 ; K O, n=6$ ). ${ }^{\star} P<0.05$ by Student's $t$ test; ${ }^{*} P<0.05$ by two-way ANOVA. Light blue bars represent $p 110 \alpha-f l o x e d$ mice;

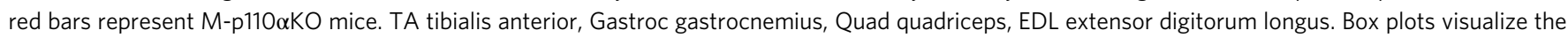
five-number summary of the data set (minimum, lower quartile, median, upper quartile, and maximum). The data are mean \pm SEM 
glucose tolerance and insulin sensitivity were not different between M-p110aKO and controls, we assessed in vivo glucose uptake specifically in muscle and adipose tissue utilizing tritiated glucose. Interestingly, despite impaired insulin signaling in $\mathrm{M}$ p1 $10 \alpha \mathrm{KO}$, there was no significant difference in basal or insulinstimulated glucose uptake in TA and soleus muscle in controls and M-p110aKO (Supplementary Fig. 5f). Likewise, as expected, M-p110ßKO as compared with controls at 3 months of age had no difference in GTT and ITT, both on CD and HFD, although all mice showed higher glucose levels throughout GTT and ITT on HFD (Supplementary Fig. 5g, h).

Decreased muscle mass, fiber size, and increased myogenesis. At 3 months of age, M-p110aKO displayed a 15\% decrease of body weight compared with controls (Supplementary Fig. 6a). This was due, in large part, to $12-24 \%$ reductions of mass in most muscles (all $p<0.05$, Student's $t$ test) (Supplementary Fig. 6b), except soleus muscle, whose weight was not different between the groups. By contrast, no difference was observed in the body or muscle weight of M-p110ßKO at 3 months of age compared with controls (Supplementary Fig. 6c, d). The reduced muscle mass in M-p110aKO was due to a $30-40 \%$ decrease of the muscle fiber cross-sectional area as quantitated using hematoxylin and eosin (H\&E) and succinate dehydrogenase $(\mathrm{SDH})$ stained sections (Fig. 6d; Supplementary Fig. 6e). There was also a $48 \%$ increase in the muscle fiber number in crosssections of quadriceps muscles, and a ten-fold increase in myofibers with central nuclei in $\mathrm{M}-\mathrm{p} 110 \mathrm{aKO}(10 \%$ vs. $1 \%$ in controls) (Fig. 6d), indicating increased myogenesis in these mice $^{36}$. SDH staining of TA muscles showed a preponderance of glycolytic fibers with no difference between controls and $\mathrm{M}$ p110aKO (Supplementary Fig. 6f, g). Consistent with increased myogenesis, there was increased expression of MyoD1 (49\%), Myf5 (50\%), and myogenin (39\%) in TA muscle of 3-month-old M-p110aKO (all $p<0.05$, Student's $t$ test) (Fig. 6e).

Expression of myostatin, a TGF $\beta$ family member that has an inhibitory role in muscle growth ${ }^{37}$, on the other hand, was decreased by $44 \%$ in muscle of M-p110aKO (Fig. 6e). The decrease in myostatin and increase in MyoD1 were also observed even after 24 -h fasting (Supplementary Fig. 6h), when protein catabolism of muscle is increased ${ }^{38}$. Previously we have generated mice with a heterozygous knockin mutation changing Arg 649 to Trp in the gene for the p85a PI3K regulatory subunit. This results in significantly decreased insulin signaling ${ }^{39}$. This was also associated with decreased myostatin expression and a tendency for increased MyoD1 mRNA, thus providing further evidence that the impaired PI3K signaling results in decreased myostatin expression (Supplementary Fig. 6i).

The decrease in myostatin mRNA in M-p110aKO was associated with a parallel reduction of serum myostatin levels in fed and fasted states (Fig. 6f). Reduction in circulating myostatin has been associated with increased muscle mass and decreased fat mass $^{40}$. In M-p110aKO, there was a $35 \%$ decrease in weight of the epididymal fat pad, and a $28 \%$ decrease in weight of the subcutaneous fat pad compared with controls $(p<0.05$, Student's $t$ test) (Fig. 6g). The decreased adipose tissue mass was associated with increased expression of genes involved in lipolysis, including $\sim 70 \%$ increases in mRNA of adipocyte triglyceride lipase (ATGL) and hormone-sensitive lipase (HSL) (Supplementary Fig. 6j). Likewise, mRNA levels of genes that regulate $\beta$-oxidation were increased in adipose tissue of $\mathrm{M}$-p110aKO, including peroxisome proliferator-activated receptor- $\alpha$ (PPAR $\alpha$ ) (145\%), PGC1 $\alpha 1$ (341\%), very-long chain acyl-CoA dehydrogenase (VLCAD) $(48 \%)$, and long-chain acyl-CoA dehydrogenase (LCAD) (62\%) $(p<0.05$, Student's $t$ test) (Supplementary Fig. 6k).
The combined reduction in muscle and fat mass was associated with a $76 \%$ increase in triglyceride accumulation in muscle of $\mathrm{M}$ p110aKO in fed state, and a margin $34 \%$ increase in the fasted state (Fig. 6h). This was associated with a $60 \%$ increase in expression of fatty acid transport protein 1 (FATP1), the major transporter for fatty acid uptake in muscle of M-p110aKO (Supplementary Fig. 7a) ${ }^{41}$. These changes occurred with no changes in mRNA expression of genes involved in lipolysis in muscle of M-p110aKO (Supplementary Fig. 7b).

Abnormal muscle function and decreased RER in M-p110aKO. While no functional defect in endurance or strength was present in M-p110aKO at 3 months of age (Supplementary Fig. 7c, d), by 16 months of age M-p110aKO exhibited a 33\% decrease of exercise capacity on a treadmill test $(p=0.07)$ (Fig. 6i) and a $16 \%$ decrease of muscle grip strength ( $p<0.05$, Student's $t$ test) (Fig. $6 \mathrm{j}$, Student's $t$ test). Likewise, in CLAMS metabolic cage assessment at 3 months of age, M-p110aKO displayed similar food intake, activity, $\mathrm{O}_{2}$ consumption, $\mathrm{CO}_{2}$ production, and respiratory exchange ratio (RER), versus controls in both fed and fasted conditions (Supplementary Fig. 7e-i). However, by 24 months of age, fasted M-p110aKO exhibited $47-63 \%$ decreases in spontaneous activity compared with controls, as assessed in CLAMS metabolic cages (Fig. 6k). Food intake, adjusted for body weight, was not different between M-p110aKO and controls at 24 months of age (Supplementary Fig. 7j). RER was decreased by $9 \%$ in $\mathrm{M}$ p110aKO during the light cycle under fed conditions $(0.74 \pm 0.01$ vs. $0.81 \pm 0.023, p<0.05$, Student's $t$ test) (Fig. 61 ), due to a $13 \%$ of decrease in $\mathrm{CO}_{2}$ production in $\mathrm{M}-\mathrm{p} 110 \alpha \mathrm{KO}$ (Fig. $6 \mathrm{~m}$ ), indicating a shift of energy utilization from carbohydrate toward fat. Oxygen consumption was not different between M-p110aKO and controls in either fed or fasted conditions (Supplementary Fig. 7k). Together, these data suggest a shift of energy utilization from carbohydrate consumption toward fatty acid oxidation in aged M-p110aKO mice, accompanied by decreased spontaneous activity and exercise capacity.

\section{Discussion}

PI3K and its product PIP3 are the central signaling nodes in insulin regulation of metabolism ${ }^{1,12,42,43}$. In this study, we have investigated the roles of two major isoforms of the PI3K catalytic subunit, p1 $10 \alpha$ and $\mathrm{p} 110 \beta$, in regulation of metabolism in skeletal muscle. Similar to the liver, we find that a loss of p110a, but not p1 $10 \beta$, in muscle leads to impaired insulin signaling, as evidenced by diminished phosphorylation of its downstream target AKT. This difference between a loss of p110 $\alpha$ and $\mathrm{p} 110 \beta$ can be explained, at least in part, by different gene expression, as $p 110 \alpha$ mRNA is 12 -fold higher than $p 110 \beta$ in muscle. In addition, it is possible that $\mathrm{p} 110 \alpha$ and $\mathrm{p} 110 \beta$ have different intrinsic functions as suggested by our data and previous studies ${ }^{2,3}$.

Although deletion of $\mathrm{p} 110 \alpha$, but not $\mathrm{p} 110 \beta$, results in impaired muscle insulin signaling and hyperinsulinemia, these mice had normal whole-body glucose tolerance and insulin sensitivity. Furthermore, glucose uptake in skeletal muscle is also not impaired in M-p110aKO as measured in vivo by radioactively labeled glucose. Likewise, glucose tolerance and insulin sensitivity are not different even after 13 weeks on HFD or with aging, suggesting that in vivo, the defect in insulin signaling produced by a loss of p110a in muscle only is not sufficient to impair glucose tolerance.

Normal whole-body glucose tolerance in M-p110aKO is consistent with our findings of normal glucose tolerance in mice with muscle-specific deletion of insulin receptor ${ }^{44}$ or insulin and IGF1 receptors ${ }^{10}$. The latter has a complete lack of insulin signaling, but an increase in non-insulin-mediated glucose uptake, 
secondary to decreased TBC1D1 resulting in increased Glut4 membrane localization ${ }^{10}$. Whether this is true in muscle of $\mathrm{M}$ p110aKO remains to be determined. Along the same line, muscle-specific deletion of the regulatory $\mathrm{p} 85 \mathrm{a}$ subunit of PI3K, which destabilizes and reduces p110a protein, does not result in glucose intolerance ${ }^{43}$. By contrast, work from our lab and others have shown that mice with muscle-specific knockout of Glut4, which is responsible for both insulin-mediated and exerciseinduced glucose uptake, develop markedly impaired glucose tolerance $^{45}$, indicating that muscle glucose uptake, when completely impaired, is important in whole body glucose homeostasis.

Despite a lack of obvious defects in glucose metabolism, a loss of p110a does induce fasting-like phenotype in muscle characterized by increased mitochondrial function and fatty acid oxidation. This is evident by increased levels of electron transport chain complex proteins and enhanced expression and enzymatic activity of citrate synthase in muscle of M-p110aKO. There is also an eightfold increase in mitochondrial NADH levels, an increase in cellular ATP levels, and increased $\mathrm{C}^{14}$-labeled palmitate oxidation in the isolated mitochondria. However, this shift toward increased fatty acid oxidation comes at a cost of higher ROS levels. Loss of $\mathrm{p} 110 \mathrm{\alpha}$ is also associated with increased proteasome and autophagy-lysosomal muscle degradation, reduced muscle mass, and smaller muscle fibers, especially in glycolytic muscles, whereas predominantly oxidative soleus muscle appears to adapt to higher fatty acid oxidation. These findings are in line with some clinical studies showing increased mitochondrial activity and fatty acid oxidation with the development of muscle insulin resistance in T2DM and obesity ${ }^{6}$.

Increased mitochondrial activity in M-p110aKO is likely due to increased expression of $P G C 1 \alpha$ isoforms. Indeed, as a major regulator of mitochondrial biogenesis, mice overexpressing PGC1 $\alpha$ have more newly formed muscle fibers and an increase in mitochondrial mass ${ }^{46}$, both features of M-p110aKO. Mitochondrial fusion, a process that promotes ATP production, is increased in $\mathrm{M}-\mathrm{p} 110 \alpha \mathrm{KO}$, as a result of increased expression of regulators of mitochondrial fusion such as Mfn2. This process may also be regulated by the increased PGC1 $\alpha$ in M-p110aKO ${ }^{47}$. Other studies have shown a strong correlation between intramuscular lipid storage and increased PGC1 $\alpha$ expression ${ }^{48}$. This is also true in M-p110aKO mouse muscle, which has increased triglyceride accumulation. Indeed, many studies have shown that several weeks of HFD treatment in rodents induces an increase in mitochondrial biogenesis in skeletal muscle ${ }^{8}$. This elevated fatty acid influx could induce an adaptive increase in mitochondrial biogenesis and function in muscle, as a way to achieve energy homeostasis. However, the magnitude of these changes in mitochondria appears to be insufficient to overcome enhanced lipid availability, therefore ectopic lipid accumulation is observed in $\mathrm{M}$-p110aKO. Another interesting aspect of these changes is that the increase in $P G C \alpha 1$ occurs primarily in two of the less studied isoforms, $P G C 1 \alpha 2$ and $P G C 1 \alpha 3$, and how these differ from the effects of the better characterized $P G C 1 \alpha 1$ and $P G C 1 \alpha 4$ remains to be determined.

In any case, the loss of p110a results in increased mitochondrial biogenesis and function. This appears to be a compensatory mechanism secondary to a decrease in insulin signaling and glycolytic capacity. This is evident by higher levels of ROS-a byproduct of mitochondrial respiration-in muscle of M-p110aKO. Previous studies have shown that ROS produced by the mitochondria can stabilize LC3, the key factor in autophagosome biogenesis, and therefore is essential for stress-induced autophagy ${ }^{49,50}$. Indeed, high levels of ROS have been shown to increase proteolysis and decrease protein synthesis, contributing to the pathogenesis of muscle atrophy ${ }^{51}$. In line with this, increased autophagy signaling is displayed in $\mathrm{M}-\mathrm{p} 110 \mathrm{\alpha KO}$ along with decreased skeletal muscle mass. Interestingly, mitochondrial size, LC3-mediated autophagy, increased proteolyis, and decreased muscle mass were only evident in glycolytic and mixed muscle fibers, but not in oxidative muscle such as soleus, which is able to adapt better to fatty acid oxidation.

In $\mathrm{M}-\mathrm{p} 110 \mathrm{aKO}$, there is also decreased circulating myostatin. This results in increases in myoblast proliferation, numbers of muscle fibers per muscle, and numbers of immature muscle fibers with central nuclei ${ }^{52}$. This increased myogenesis is a compensatory mechanism secondary to increased muscle protein degradation, such that muscle mass is reduced by only $10-15 \%$ in $\mathrm{M}$ p1 $10 a \mathrm{KO}$, despite the relatively large changes in each of the individual processes controlling maintenance of muscle mass. Interestingly, a decrease in PI3K signaling in M-p110aKO associated with a decrease in muscle expression of myostatin is also evident in our mice with dominant-negative mutation in the p85a subunit of PI3K, suggesting a direct role of PI3K in muscle homeostasis. This finding raises concern that prolonged use of PI3K inhibitors in cancer patients, especially those targeted against $\mathrm{p} 110 \mathrm{a}^{53}$, may add to muscle atrophy already present in many of these individuals.

Whereas decreased myostatin levels were not sufficient to overcome reduced muscle size in $\mathrm{M}$-p1 $10 \alpha \mathrm{KO}$, it could account for increased lipolysis and reduced whole-body fat mass, contributing to relatively mild metabolic impairment. Indeed, previous studies have shown that inhibition of myostatin action or myostatin deficiency results in increased muscle mass accompanied by decreased fat mass ${ }^{54}$. M-p110aKO have $40-50 \%$ decreases of myostatin expression in muscle and serum myostatin. This may contribute to increased adipose tissue lipolysis through increased expression of ATGL and HSL in adipose tissue $^{55}$. In $\mathrm{M}-110 \mathrm{aKO}$, there is also an increase in FATP1 in muscle, which could lead to increased lipid uptake by muscle ${ }^{56}$, decreased adipose tissue mass, and accumulation of triglycerides in muscle of M-p110aKO, a phenotype found in some models of muscle atrophy ${ }^{57}$. Myostatin inhibition is also one possible driver for increased PGC1a expression in M-p110aKO. Indeed, previous studies have shown PGC1a expression is induced in myostatin inhibition models ${ }^{58}$, and in line with this, M-p110aKO display increased mRNA expression of PGC1 $\alpha$ in both skeletal muscle and white adipose tissue, suggesting a systemic regulation of PGC1 $\alpha$ gene expression.

In summary, our study demonstrates that p110a is the dominant catalytic isoform of PI3K in muscle and plays a unique role in metabolic regulation. p110a is critical for the maintenance of muscle mass. It also plays an important and unexpected role in the regulation of mitochondrial homeostasis. Deletion of p110a in muscle leads to significant increase in proteasome activity and autophagy, resulting in reduction of muscle mass, strength, and endurance, especially with age. Interestingly, loss of p110a in skeletal muscle causes an increase, rather than decrease, in mitochondrial metabolism, as evidenced by increases in mitochondrial biogenesis, mitochondrial fusion, oxidative capacity, and levels of ROS. This is likely due to increased expression of $P G C 1 \alpha$ isoforms found in M-p110aKO. Thus, changes at the level of p110a may help explain differences in muscle mitochondrial function in the context of diabetes. These data shed light on how p110a regulates metabolic functions in skeletal muscle and add a new layer of understanding the relationship between mitochondrial function and insulin sensitivity.

\section{Methods}

Animal care and use. All studies were performed in male mice on $C 57 B L / 6 \mathrm{~J}$ background. Muscle-specific p110a or p110 $\beta$ knockout mice were generated by crossing mice carrying the Cre recombinase gene driven by the human alphaskeletal actin (HSA) promoter (Jackson Laboratories Stock Number: 006149) with 
mice carrying either floxed $\mathrm{p} 110 \alpha$ or $\mathrm{p} 110 \beta$ alleles, in which exon 1 of $\mathrm{p} 110 \alpha$ or exon 2 of $\mathrm{p} 110 \beta$ was flanked with loxP sites ${ }^{2,3,59}$. All animal studies were approved by the Institutional Animal Care and Use Committee (IACUC) at the Joslin Diabetes Center, and were in accordance with the National Institutes of Health guidelines. See Supplementary Methods for more information.

RNA-Seq processing and data analysis. RNA-Seq analysis was performed as previously reported ${ }^{60}$. Library for RNA-Seq was prepared using the NEBNext mRNA Sample Prep Master Mix kit (NEB), and HTG EdgeSeq mRNA sequence analysis was performed by BioPolymers Facility at Harvard Medical School. The total RNA was extracted from tissues using Trizol and was reverse-transcribed using a high-capacity complementary DNA reverse transcription kit (Applied Biosystems) according to the manufacturer's instructions. Quantitative real-time PCR was performed in $5 \mu \mathrm{l}$ of the resulting CDNA after a tenfold dilution in the presence of the SYBR Green PCR Master Mix (Applied Biosystems) and $300 \mathrm{nM}$ primers. PCR reactions were run in duplicate in the ABI Prism 7700 Sequence Detection System with primers as detailed in Supplementary Table 1, and Ct values were normalized to GAPDH gene levels. KEGG enrichment analysis was performed using a hypergeometric distribution test. Filtering of gene expression and statistics were performed using MATLAB 2016b.

Proteolysis assay. Proteolysis was measured as previously described ${ }^{61}$. Briefly, EDL and soleus muscles were isolated from M-p110aKO and controls, and preincubated in $1 \mathrm{ml}$ of KRB buffer ( $117 \mathrm{mM} \mathrm{NaCl}, 4.7 \mathrm{mM} \mathrm{KCl}, 2.5 \mathrm{mM} \mathrm{CaCl}_{2}, 1.2$ $\mathrm{mM} \mathrm{KH} \mathrm{PO}_{4}, 1.2 \mathrm{mM} \mathrm{MgSO}_{4}, 24.6 \mathrm{mM} \mathrm{NaHCO}_{3}$, and $5 \mathrm{mM}$ glucose) for $30 \mathrm{~min}$ before transferring to fresh $1 \mathrm{ml}$ of $\mathrm{KRB}$ containing $0.5 \mathrm{mM}$ cycloheximide to inhibit protein synthesis. Incubation buffer was collected, and tyrosine release was measured 62

Proteasome measurement. Proteasome activity was measured from gastrocnemius muscles of M-p110aKO and controls fasted as previously described ${ }^{15}$. Briefly, muscle fractions containing proteasome were isolated from homogenized muscle lysates after centrifugation. A peptidyl glutamyl-like (LLE) substrate (ZLeu-Leu-Glu-7-amido-4-methylcoumarin) (Sigma-Aldrich, C0483) was added into assay buffer, and fluorescence was monitored (360 - nm excitation, $460-\mathrm{nm}$ emission) every $3 \mathrm{~min}$ for $1.5 \mathrm{hrs}$ at $37^{\circ} \mathrm{C}$. Enzyme activity of $26 \mathrm{~S}$ proteasome was determined as the change in fluorescence during the linear phase of the reaction and compared against a standard curve of 7-amido-4-methylcoumarin (SigmaAldrich, A9891).

Mitochondrial respiratory enzyme activities. Mitochondrial assays were performed according to established methods ${ }^{63}$. The NADH cytochrome $c$ reductase (NCCR) and succinate cytochrome $c$ reductase (SCCR) activities were measured by following the reduction of exogenous oxidized cytochrome $c$. An aliquot of $10 \mu \mathrm{g}$ of isolated mitochondria was preincubated with the assay buffer $(1.5 \mathrm{mM} \mathrm{KCN}, 50$ $\mathrm{mM} \mathrm{K}_{2} \mathrm{HPO}_{4}$, pH 7.4) containing $\beta$-NADH or succinate at $37^{\circ} \mathrm{C}$ for $15 \mathrm{~min}$. After addition of cytochrome $c$ to the mixture, the change in the absorbance at $550 \mathrm{~nm}$ was recorded on a spectrophotometer. Cytochrome $c$ oxidase (CCO) activity was determined by following the oxidation of exogenous reduced cytochrome $c$. An aliquot of $5 \mu \mathrm{g}$ of isolated mitochondria was preincubated in the assay buffer ( 5 $\mathrm{mM} \mathrm{K} \mathrm{HPO}_{4}, \mathrm{pH} \mathrm{7.4)}$ at $37^{\circ} \mathrm{C}$ for $10 \mathrm{~min}$. After addition of ferrocytochrome $c$ to the assay mixture, the change in absorbance at $550 \mathrm{~nm}$ was recorded on a spectrophotometer.

EM and morphometric analysis. After perfusion with PBS, soleus and EDL muscles were dissected from mice and fixed using $2.5 \%$ (wt/vol) glutaraldehyde in $0.1 \mathrm{M}$ phosphate buffer for $4 \mathrm{hrs}$ at room temperature before being placed at $4{ }^{\circ} \mathrm{C}$ for storage. Sample preparation for EM was performed by EM Core Facility of Harvard Medical School ${ }^{64}$. After being placed in ascending concentrations of ethanol, samples were incubated in 1:1 mixture of propylene oxide and Araldite 502 epoxy resin for several hours before being placed into a vacuum desiccator overnight. This was followed by embedding samples in Araldite 502 epoxy resin using BEEM capsules (Ted Pella Inc.) and a $60^{\circ} \mathrm{C}$ curing oven for $48 \mathrm{hrs}$. Sections were trimmed and adhered to glass slides using heat, stained with a $1 \%$ methylene blue, and prepared for EM. Morphological analysis such as mitochondrial size and area was obtained using Metamorph software (v 6.1, Universal imaging) ${ }^{34}$. At least four sections were measured per animal in a double-blind manner.

Plasmid transfection and intravital microscopy. For in vivo imaging, mice were anesthetized and mounted on the microscope stage on their side in dental cement in the position to expose the tissue to be imaged ${ }^{22}$. Images were collected with a $63 \times, 1.2$ NA Zeiss C-Apochromat objective on a Zeiss-LSM-710 microscope. MitoGFP was excited using the 488 -nm laser line. Mito-EGFP emission was collected at $505-576 \mathrm{~nm}$. MitoTimer was excited using the 488 (green) and 561 -nm laser line (red). MitoTimer emissions were collected at $505-540 \mathrm{~nm}$ (green channel) and $590-640 \mathrm{~nm}$ (red channel). NADH was excited and collected as previously described ${ }^{64}$.
Fatty acid oxidation by isolated mitochondria. Mitochondria were isolated and purified from hindlimb muscle of M-p110 $\alpha$ and controls as previous described ${ }^{13}$ and fatty acid oxidation was measured by the conversion of $\left[1-{ }^{14} \mathrm{C}\right]$-palmitate acid into $\mathrm{CO}_{2}$ in isolated mitochondria ${ }^{65}$. Briefly, isolated mitochondria were placed in a buffer containing BSA-conjugated $\left[1-{ }^{14} \mathrm{C}\right]$-palmitate acid for $1 \mathrm{~h}$ at $37^{\circ} \mathrm{C} . \mathrm{CO}_{2}$ was released from buffer by addition of $70 \%$ perchloric acid, and was captured in 3 $\mathrm{M} \mathrm{NaOH}$ that had been added to a filter paper on top of the vial. Fatty acid oxidation was calculated based on the level of ${ }^{14} \mathrm{CO}_{2}$ trapped in the filter paper by scintillation counting.

In vivo glucose uptake. Glucose uptake was measured as described ${ }^{66}$. In brief, glucose uptake into tissue was measured by intravenous injection of $0.33 \mu \mathrm{Ci}$ of $\mathrm{H}^{3}$ labeled glucose per gram of body weight dissolved in either saline or $20 \%$ dextrose, administered via the retro-orbital sinus. After $25 \mathrm{~min}$, disintegrations per min were measured in selected tissue and in blood at $0,5,10$, and $15 \mathrm{~min}$.

Oxygen consumption rate analysis. Mitochondria were isolated and purified from hindlimb muscle, then diluted in the Mitochondrial Assay Solution supplied with $10 \mathrm{mM}$ pyruvate $/ 2 \mathrm{mM}$ malate as previously described ${ }^{13}$. Oxygen consumption rate (OCR) was measured using a Seahorse Bioscience XF24 analyzer in the absence (basal) or presence (state 3) of $400 \mu \mathrm{M}$ adenosine diphosphate (ADP). Protein concentrations were measured using the Bradford method (Bio-Rad).

Western blot analysis. Powdered muscle tissue was homogenized in RIPA buffer (Millipore, 20-188) supplemented with phosphatase inhibitor and protease inhibitor cocktail (Sigma-Aldrich). Lysates were subjected to SDS-PAGE and blotted using following antibodies: p110a (CS4249, 1:1000), phospho-Akt (S473) (CS9271, 1:2000), phospho-ERK (CS9101, 1:2000), phospho-GSK3 $\beta$ (CS9336, 1:2000), phospho-4EBP1 (CS2855, 1:1000), phospho-ULK (S555) (CS5869, 1:1000), phospho-Drp1 (S637) (CS4867, 1:1000), phospho-FoxO1/3 (CS9464, 1:500), ERK (CS9102, 1:2000), GSK3 3 (CS9315, 1:2000), 4EBP1(CS9644, 1:500), LC3 (CS2775 1:1000), Beclin-1(CS3495, 1:1000), Drp1 (CS8570, 1:1000) and FoxO1 (CS2880, 1:500) from Cell Signaling, OPA1 (SC393296, 1:1000), GAPDH from Santa Cruz (SC25778, 1:5000), and citrate synthase (Ab96600, 1:1000), Mfn2 (Ab56889, 1:1000), and OXOPHOS (Ab110413, 1:1000) proteins from Abcam. Uncropped blots are available in Supplementary Fig. 8.

Statistical analysis. The data are expressed as mean \pm SEM. Differences were analyzed using an unpaired Student's $t$ test, One-way ANOVA or two-way ANOVA as appropriate. One-way ANOVA was followed by the Tukey's multiple comparison post hoc test, and two-way ANOVA was performed using Bonferroni's multiple comparison post test. Statistical calculations were performed using the GraphPad Prism software (GraphPad, San Diego, CA). A probability value of $<0.05$ was considered significantly different.

Reporting summary. Further information on research design is available in the Nature Research Reporting Summary linked to this article.

\section{Data availability}

All relevant data are included in the article and Supplementary Information. The RNAseq data generated in this study are available at NCBI GEO database with the accession number GS E124394. Additional data generated during and/or analyzed during this study are available from the corresponding author on reasonable request.

Received: 6 December 2017 Accepted: 2 July 2019

Published online: 30 July 2019

\section{References}

1. Taniguchi, C. M. et al. Divergent regulation of hepatic glucose and lipid metabolism by phosphoinositide 3-kinase via Akt and PKClambda/zeta. Cell Metab. 3, 343-353 (2006)

2. Sopasakis, V. R. et al. Specific roles of the p110alpha isoform of phosphatidylinsositol 3-kinase in hepatic insulin signaling and metabolic regulation. Cell Metab. 11, 220-230 (2010).

3. Jia, S. et al. Essential roles of PI(3)K-p110beta in cell growth, metabolism and tumorigenesis. Nature 454, 776-779 (2008).

4. Nelson, V. L., Jiang, Y. P., Dickman, K. G., Ballou, L. M. \& Lin, R. Z. Adipose tissue insulin resistance due to loss of PI3K p110alpha leads to decreased energy expenditure and obesity. Am. J. Physiol. Endocrinol. Metab. 306, E1205-E1216 (2014).

5. DeFronzo, R. A. \& Tripathy, D. Skeletal muscle insulin resistance is the primary defect in type 2 diabetes. Diabetes Care 32(Suppl 2), S157-S163 (2009). 
6. Hesselink, M. K., Schrauwen-Hinderling, V. \& Schrauwen, P. Skeletal muscle mitochondria as a target to prevent or treat type 2 diabetes mellitus. Nat. Rev. Endocrinol. 12, 633-645 (2016).

7. Trenell, M. I., Hollingsworth, K. G., Lim, E. L. \& Taylor, R. Increased daily walking improves lipid oxidation without changes in mitochondrial function in type 2 diabetes. Diabetes Care 31, 1644-1649 (2008).

8. Turner, N. et al. Excess lipid availability increases mitochondrial fatty acid oxidative capacity in muscle: evidence against a role for reduced fatty acid oxidation in lipid-induced insulin resistance in rodents. Diabetes $\mathbf{5 6}$, 2085-2092 (2007).

9. Park, S. W. et al. Decreased muscle strength and quality in older adults with type 2 diabetes: the health, aging, and body composition study. Diabetes 55, 1813-1818 (2006).

10. O’Neill, B. T. et al. Differential role of insulin/IGF-1 receptor signaling in muscle growth and glucose homeostasis. Cell Rep. 11, 1220-1235 (2015).

11. Kim, Y. B., Nikoulina, S. E., Ciaraldi, T. P., Henry, R. R. \& Kahn, B. B. Normal insulin-dependent activation of Akt/protein kinase B, with diminished activation of phosphoinositide 3-kinase, in muscle in type 2 diabetes. J. Clin. Invest. 104, 733-741 (1999).

12. Cusi, K. et al. Insulin resistance differentially affects the PI 3-kinase- and MAP kinase-mediated signaling in human muscle. J. Clin. Invest. 105, 311-320 (2000).

13. Li, M., Vienberg, S. G., Bezy, O., O’Neill, B. T. \& Kahn, C. R. Role of PKCdelta in insulin sensitivity and skeletal muscle metabolism. Diabetes 64, 4023-4032 (2015).

14. Milan, G. et al. Regulation of autophagy and the ubiquitin-proteasome system by the FoxO transcriptional network during muscle atrophy. Nat. Commun. 6, 6670 (2015).

15. O’Neill, B. T. et al. Insulin and IGF-1 receptors regulate FoxO-mediated signaling in muscle proteostasis. J. Clin. Invest. 126, 3433-3446 (2016).

16. Mizushima, N., Yoshimori, T. \& Levine, B. Methods in mammalian autophagy research. Cell 140, 313-326 (2010).

17. Baumeister, W., Walz, J., Zuhl, F. \& Seemuller, E. The proteasome: paradigm of a self-compartmentalizing protease. Cell 92, 367-380 (1998).

18. Nakae, J., Kitamura, T., Silver, D. L. \& Accili, D. The forkhead transcription factor Foxol (Fkhr) confers insulin sensitivity onto glucose-6-phosphatase expression. J. Clin. Invest. 108, 1359-1367 (2001).

19. Sandri, M. et al. Foxo transcription factors induce the atrophy-related ubiquitin ligase atrogin-1 and cause skeletal muscle atrophy. Cell 117, 399-412 (2004).

20. Mammucari, C. et al. FoxO3 controls autophagy in skeletal muscle in vivo. Cell Metab. 6, 458-471 (2007).

21. Liesa, M., Palacin, M. \& Zorzano, A. Mitochondrial dynamics in mammalian health and disease. Physiol. Rev. 89, 799-845 (2009).

22. Lauritzen, H. P. et al. Gene gun bombardment-mediated expression and translocation of EGFP-tagged GLUT4 in skeletal muscle fibres in vivo. Pflug. Arch. 444, 710-721 (2002).

23. Liesa, M. \& Shirihai, O. S. Mitochondrial dynamics in the regulation of nutrient utilization and energy expenditure. Cell Metab. 17, 491-506 (2013).

24. Cipolat, S., Martins de Brito, O., Dal Zilio, B. \& Scorrano, L. OPA1 requires mitofusin 1 to promote mitochondrial fusion. Proc. Natl Acad. Sci. USA 101, 15927-15932 (2004)

25. Frank, S. et al. The role of dynamin-related protein 1 , a mediator of mitochondrial fission, in apoptosis. Dev. Cell 1, 515-525 (2001).

26. Romanello, V. et al. Mitochondrial fission and remodelling contributes to muscle atrophy. EMBO J. 29, 1774-1785 (2010).

27. Shepherd, D. \& Garland, P. B. The kinetic properties of citrate synthase from rat liver mitochondria. Biochem. J. 114, 597-610 (1969).

28. Besseiche, A., Riveline, J. P., Gautier, J. F., Breant, B. \& Blondeau, B. Metabolic roles of PGC-1alpha and its implications for type 2 diabetes. Diabetes Metab. 41, 347-357 (2015).

29. Millay, D. P. \& Olson, E. N. Making muscle or mitochondria by selective splicing of PGC-1alpha. Cell Metab. 17, 3-4 (2013).

30. Ruas, J. L. et al. A PGC-1alpha isoform induced by resistance training regulates skeletal muscle hypertrophy. Cell 151, 1319-1331 (2012).

31. Rothstein, E. C., Carroll, S., Combs, C. A., Jobsis, P. D. \& Balaban, R. S. Skeletal muscle NAD(P)H two-photon fluorescence microscopy in vivo: topology and optical inner filters. Biophys. J. 88, 2165-2176 (2005)

32. Crescenzo, R. et al. Subsarcolemmal and intermyofibrillar mitochondrial responses to short-term high-fat feeding in rat skeletal muscle. Nutrition $\mathbf{3 0}$, 75-81 (2014)

33. Laker, R. C. et al. A novel MitoTimer reporter gene for mitochondrial content, structure, stress, and damage in vivo. J. Biol. Chem. 289, 12005-12015 (2014).

34. Lauritzen, H. P. et al. Contraction and AICAR stimulate IL-6 vesicle depletion from skeletal muscle fibers in vivo. Diabetes 62, 3081-3092 (2013).

35. Fink, R. I., Kolterman, O. G., Griffin, J. \& Olefsky, J. M. Mechanisms of insulin resistance in aging. J. Clin. Invest. 71, 1523-1535 (1983).
36. Jarvinen, T. A., Jarvinen, M. \& Kalimo, H. Regeneration of injured skeletal muscle after the injury. Muscles, Liga. tendons J. 3, 337-345 (2013).

37. McPherron, A. C., Lawler, A. M. \& Lee, S. J. Regulation of skeletal muscle mass in mice by a new TGF-beta superfamily member. Nature $\mathbf{3 8 7}, 83-90$ (1997).

38. Jeanplong, F. et al. Prolonged underfeeding of sheep increases myostatin and myogenic regulatory factor Myf-5 in skeletal muscle while IGF-I and myogenin are repressed. J. Endocrinol. 176, 425-437 (2003).

39. Winnay, J. N. et al. PI3-kinase mutation linked to insulin and growth factor resistance in vivo. J. Clin. Invest. 126, 1401-1412 (2016).

40. Bostrom, P. et al. A PGC1-alpha-dependent myokine that drives brown-fatlike development of white fat and thermogenesis. Nature 481, 463-468 (2012)

41. Kim, J. K. et al. Inactivation of fatty acid transport protein 1 prevents fatinduced insulin resistance in skeletal muscle. J. Clin. Invest. 113, 756-763 (2004).

42. Dowell, P., Otto, T. C., Adi, S. \& Lane, M. D. Convergence of peroxisome proliferator-activated receptor gamma and Foxol signaling pathways. J. Biol. Chem. 278, 45485-45491 (2003).

43. Luo, J. et al. Loss of class IA PI3K signaling in muscle leads to impaired muscle growth, insulin response, and hyperlipidemia. Cell Metab. 3, 355-366 (2006).

44. Bruning, J. C. et al. A muscle-specific insulin receptor knockout exhibits features of the metabolic syndrome of NIDDM without altering glucose tolerance. Mol. Cell 2, 559-569 (1998).

45. Zisman, A. et al. Targeted disruption of the glucose transporter 4 selectively in muscle causes insulin resistance and glucose intolerance. Nat. Med. 6, 924-928 (2000).

46. Hoeks, J. et al. Enhanced lipid-but not carbohydrate-supported mitochondrial respiration in skeletal muscle of PGC-1alpha overexpressing mice. J. Cell Physiol. 227, 1026-1033 (2012).

47. Soriano, F. X. et al. Evidence for a mitochondrial regulatory pathway defined by peroxisome proliferator-activated receptor-gamma coactivator-1 alpha, estrogen-related receptor-alpha, and mitofusin 2. Diabetes 55, 1783-1791 (2006).

48. Haemmerle, G. et al. ATGL-mediated fat catabolism regulates cardiac mitochondrial function via PPAR-alpha and PGC-1. Nat. Med. 17, 1076-1085 (2011).

49. Le Lay, S., Lefrere, I., Trautwein, C., Dugail, I. \& Krief, S. Insulin and sterolregulatory element-binding protein-1c (SREBP-1C) regulation of gene expression in 3T3-L1 adipocytes. Identification of CCAAT/enhancer-binding protein beta as an SREBP-1C target. J. Biol. Chem. 277, 35625-35634 (2002).

50. Yang, Q., Bassel-Duby, R. \& Williams, R. S. Transient expression of a wingedhelix protein, MNF-beta, during myogenesis. Mol. Cell Biol. 17, 5236-5243 (1997).

51. Jackson, M. J. \& McArdle, A. Age-related changes in skeletal muscle reactive oxygen species generation and adaptive responses to reactive oxygen species. $J$. Physiol. 589, 2139-2145 (2011)

52. Shi, X. et al. Foxk1 promotes cell proliferation and represses myogenic differentiation by regulating Foxo4 and Mef2. J. Cell Sci. 125, 5329-5337 (2012).

53. Zhao, W., Qiu, Y. \& Kong, D. Class I phosphatidylinositol 3-kinase inhibitors for cancer therapy. Acta Pharm. Sin. B 7, 27-37 (2017).

54. Guo, T. et al. Myostatin inhibition in muscle, but not adipose tissue, decreases fat mass and improves insulin sensitivity. PLoS One 4, e4937 (2009).

55. Zhu, H. J. et al. The effect of myostatin on proliferation and lipid accumulation in 3T3-L1 preadipocytes. J. Mol. Endocrinol. 54, 217-226 (2015).

56. Matsakas, A. et al. Investigating mechanisms underpinning the detrimental impact of a high-fat diet in the developing and adult hypermuscular myostatin null mouse. Skelet. Muscle 5, 38 (2015).

57. Krebs, J. M., Schneider, V. S., Evans, H., Kuo, M. C. \& LeBlanc, A. D. Energy absorption, lean body mass, and total body fat changes during 5 weeks of continuous bed rest. Aviat., space, Environ. Med. 61, 314-318 (1990).

58. Dong, J. et al. Inhibition of myostatin in mice improves insulin sensitivity via irisin-mediated cross talk between muscle and adipose tissues. Int J. Obes. 40, 434-442 (2016).

59. McCarthy, J. J., Srikuea, R., Kirby, T. J., Peterson, C. A. \& Esser, K. A. Inducible Cre transgenic mouse strain for skeletal muscle-specific gene targeting. Skelet. Muscle 2, 8 (2012).

60. Dong, T. G. \& Mekalanos, J. J. Characterization of the RpoN regulon reveals differential regulation of T6SS and new flagellar operons in Vibrio cholerae O37 strain V52. Nucleic Acids Res. 40, 7766-7775 (2012).

61. Price, S. R. et al. Muscle wasting in insulinopenic rats results from activation of the ATP-dependent, ubiquitin-proteasome proteolytic pathway by a mechanism including gene transcription. J. Clin. Invest. 98, 1703-1708 (1996)

62. Waalkes, T. P. \& Udenfriend, S. A fluorometric method for the estimation of tyrosine in plasma and tissues. J. Lab Clin. Med. 50, 733-736 (1957). 
63. Wang, C. H. et al. Cisd2 modulates the differentiation and functioning of adipocytes by regulating intracellular $\mathrm{Ca}^{2+}$ homeostasis. Hum. Mol. Genet. 23, $4770-4785$ (2014).

64. Kleinridders, A. et al. Leptin regulation of Hsp60 impacts hypothalamic insulin signaling. J. Clin. Invest. 123, 4667-4680 (2013).

65. Huynh, F. K., Green, M. F., Koves, T. R. \& Hirschey, M. D. Measurement of fatty acid oxidation rates in animal tissues and cell lines. Methods Enzym. 542, 391-405 (2014).

66. Softic, S. et al. Lipodystrophy due to adipose tissue-specific insulin receptor knockout results in progressive NAFLD. Diabetes 65, 2187-2200 (2016).

\section{Acknowledgements}

This work was supported by NIH grants RO1DK055545 and RO1DK033201 (to C.R.K.). M.E.L. was funded by a NIH Training Grant T32 DK007260, and B.T.O. was funded by a K08 training award from the NIDDK of the NIH (K08DK100543). The Joslin Diabetes Center DRC core facility was used for part of this work (P30 DK36836).

\section{Author contributions}

M.E.L. designed the study, researched data, and wrote the paper. H.P.M.M.L. and B.T.O. researched the data, helped design experiments, and helped write the paper. C.W., B.B., W.C., M.S., R.T. and M.F.H. researched data and helped design experiments. S.S., helped design experiments, generated additional data and revised the manuscript. C.R.K. designed the study, helped write the paper, and oversaw the research.

\section{Additional information}

Supplementary Information accompanies this paper at https://doi.org/10.1038/s41467019-11265-y.

Competing interests: The authors declare no competing interests.

Reprints and permission information is available online at http://npg.nature.com/ reprintsandpermissions/

Peer review information: Nature Communications thanks the anonymous reviewers for their contribution to the peer review of this work. Peer reviewer reports are available.

Publisher's note: Springer Nature remains neutral with regard to jurisdictional claims in published maps and institutional affiliations.

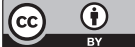

Open Access This article is licensed under a Creative Commons Attribution 4.0 International License, which permits use, sharing, adaptation, distribution and reproduction in any medium or format, as long as you give appropriate credit to the original author(s) and the source, provide a link to the Creative Commons license, and indicate if changes were made. The images or other third party material in this article are included in the article's Creative Commons license, unless indicated otherwise in a credit line to the material. If material is not included in the article's Creative Commons license and your intended use is not permitted by statutory regulation or exceeds the permitted use, you will need to obtain permission directly from the copyright holder. To view a copy of this license, visit http://creativecommons.org/ licenses/by/4.0/.

(C) The Author(s) 2019 